\title{
Spinal Wnt5a Plays a Key Role in Spinal Dendritic Spine Remodeling in Neuropathic and Inflammatory Pain Models and in the Proalgesic Effects of Peripheral Wnt3a
}

\author{
Manuela Simonetti and Rohini Kuner \\ Institute of Pharmacology, Medical Faculty Heidelberg, Heidelberg University, 69120 Heidelberg, Germany
}

Wnt signaling represents a highly versatile signaling system, which plays critical roles in developmental morphogenesis as well as synaptic physiology in adult life and is implicated in a variety of neural disorders. Recently, we demonstrated that Wnt3a is able to recruit multiple noncanonical signaling pathways to alter peripheral sensory neuron function in a nociceptive modality-specific manner. Furthermore, several studies recently reported an important role for Wnt5a acting via canonical and noncanonical signaling in spinal processing of nociception in a number of pathologic pain disorders. Here, using diverse molecular, genetic, and behavioral approaches in mouse models of pain in vivo, we report a novel role for Wnt5a signaling in nociceptive modulation at the structural level. In models of chronic pain, using male and female mice, we found that Wnt5a is released spinally from peripheral sensory neurons, where it recruits the tyrosine kinase receptors Ror2 and Ryk to modulate dendritic spine rearrangement. Blocking the Wnt5a-Ryk/Ror2 axis in spinal dorsal horn neurons prevented activity-dependent dendritic spine remodeling and significantly reduced mechanical hypersensitivity induced by peripheral injury as well as inflammation. Moreover, we observed that peripheral Wnt3a signaling triggers the release of Wnt5a in the spinal cord, and inhibition of spinal Wnt5a signaling attenuates the functional impact of peripheral Wnt3a on nociceptive sensitivity. In conclusion, this study reports a novel role for the Wnt signaling axis in coordinating peripheral and spinal sensitization and shows that targeting Wnt5a-Ryk/ROR2 signaling alleviates both structural and functional mechanisms of nociceptive hypersensitivity in models of chronic pain in vivo.

Key words: dendritic spine plasticity; neuropathic and inflammatory pain; Ror2; Ryk; spinal cord; Wnt5a

Significance Statement

There is a major need to elucidate molecular mechanisms underlying chronic pain disorders to develop novel therapeutic approaches. Wnt signaling represents a highly versatile signaling system, which plays critical roles during development and adult physiology, and it was implicated in several diseases, including chronic pain conditions. Using mouse models, our study identifies a novel role for Wnt5a signaling in nociceptive modulation at the spinal cord level. We observed that Wnt5a recruits Ror2 and Ryk receptors to enhance dendritic spine density, leading to nociceptive sensitization. Blocking the Wnt5a-Ryk/ Ror2 interaction in the spinal dorsal horn prevented spine remodeling and significantly reduced inflammatory and neuropathic hypersensitivity. These findings provide proof-of-concept for targeting spinal Wnt signaling for alleviating nociceptive hypersensitivity in vivo.

Received Dec. 8, 2019; revised Apr. 21, 2020; accepted May 18, 2020.

Author contributions: M.S. designed research; M.S. performed research; M.S. and R.K. analyzed data; M.S. and R.K. wrote the paper.

This research was supported by funding in the form of grants from the Deutsche Forschungsgemeinschaft in the Collaborative Research Center 1158 (Grant SFB1158) to M.S. (Project A09N) and R.K. (Projects B01 and B06), and partial funding from an ERC Advanced Investigator grant to R.K. (Pain Plasticity Grant 294293). R.K. is a principal investigator in the Excellence Cluster "CellNetworks" of Heidelberg University. M.S. was partly supported by an Olympia Morata Program Award from Heidelberg University. We thank the Nikon Imaging Center, Heidelberg, for help with imaging experiments.

The authors declare no competing financial interests.

Correspondence should be addressed to Manuela Simonetti at manuela.simonetti@pharma.uniheidelberg.de.

https://doi.org/10.1523/JNEUROSCI.2942-19.2020

Copyright $(2020$ the authors

\section{Introduction}

Chronic pain is one of the most prevalent health problems worldwide, as it is still poorly treated in many patients. Therefore, from a translational prospective, there is a strong need to discover new molecular mechanisms mediating the development of chronic pain to provide platforms for developing novel therapeutic strategies against nociceptive sensitization.

Wnt ligands (Wnts) are secreted glycoproteins, which act as important mediators of cell-cell communication. They are involved in diverse cellular and developmental processes such as differentiation, cell migration, and proliferation, including the development of the nervous system and synaptogenesis (Patapoutian and Reichardt, 
2000; Budnik and Salinas, 2011). Furthermore, Wnts have a crucial role in synaptic physiology, as they have the capacity to modulate synaptic transmission (Budnik and Salinas, 2011; Oliva et al., 2013a). Recent studies have demonstrated a role of Wnt signaling in postnatal brain plasticity, including memory formation and activity-regulated long-term potentiation in adult mouse brain (Oliva et al., 2013b). Dysregulation of Wnt signaling is also implicated in a variety of diseases spanning from cancer to neurologic, neurodegenerative, and psychiatric disorders, such as Alzheimer's disease and schizophrenia (Inestrosa et al., 2012; Zimmerman et al., 2012).

Wnt proteins can signal via canonical and noncanonical pathways to bring about their cellular effects. The most known Wnt-activated pathways comprise the $\mathrm{Wnt} / \beta$-catenin canonical pathway and two noncanonical pathways, namely the calcium pathway and the planar cell polarity pathway. The canonical pathway is activated when a Wnt ligand binds to Frizzled receptor and its coreceptor, namely the low-density LRP6, leading to phosphorylation of $\beta$-catenin, its stabilization in the cytosol and translocation to the nucleus, where it regulates Wnt-associated gene transcription. The noncanonical pathways are Wntdependent signaling pathways that do not affect $\beta$-catenin stabilization. The Wnt-Calcium pathway regulates intracellular calcium level targeting calcium release from the endoplasmic reticulum via phospholipase $\mathrm{C}$-inositol 1,4,5-triphosphate pathway, leading to the activation of several kinases, such as protein kinase $\mathrm{C}$, calcineurin, calcium/calmodulin-dependent protein kinase II (CaMKII) and Nemo-like kinase. The planar cell polarity pathway is activated when Wnt ligands form a ternary complex with Frizzled receptor and one of other coreceptors, namely Ryk (receptor-like tyrosine kinase), Ror2 (receptor tyrosine kinase-like orphan receptor 2), or PTK7 (protein tyrosine kinase 7). This complex recruits the small G-proteins Rho (Rho family small GTPase 1) and Rac-1 (Rac family small GTPase 1), which lead to the activation of ROCK (Rho associated coiled-coil containing protein kinase 1) and JNK (c-Jun N-terminal kinase) and cytoskeleton rearrangement. Several Wnt ligands preferentially activate either the canonical pathway or the $\beta$-catenin independent pathways. For this reason, they are traditionally divided into the following two main categories: canonical ligands, with Wnt3a being a prototype; and noncanonical ligands, with Wnt5a being a prototype. Specificity of signaling and cellular functions comes from the particular combination of Wnt ligands, Frizzled receptors, coreceptors, and even intracellular signaling components expressed and recruited in that context (Niehrs, 2012).

Recently, several research groups, including ours, uncovered a novel and important role for Wnt signaling in processes mediating hypersensitivity in chronic pain models, in both dorsal root ganglia (DRGs) and the spinal cord (Zhang et al., 2013; Itokazu et al., 2014; Simonetti et al., 2014). Indeed, while peripheral Wnt3a application is able to trigger the activation of several pathways in mouse DRG sensory neurons to elicit sensitization in inflammatory pain states (Simonetti et al., 2014), the activation of the canonical $\beta$-catenin pathway in the spinal dorsal horn was demonstrated to be important for the development of neuropathic pain in rat models (Zhang et al., 2013; Itokazu et al., 2014). Diverse Wnt ligands have been shown to be involved in the pathophysiology of pain. In particular, Wnt5a is involved in the pathogenesis of neuropathic pain associated with different origins. For example, it was shown to mediate HIV-related gp120-induced pain via JNK/TNF- $\alpha$-mediated sensitization of spinal dorsal horn neurons (Yuan et al., 2015), to contribute to the development of chronic post-thoracotomy pain, possibly by regulating noncanonical Wnt pathways the inflammatory response and nerve regeneration (Zhu et al., 2017, 2018). Wnt5a was also implicated in the pathogenesis of EAE-associated chronic pain via inducing the expression of the cytokines known mediators of neuroinflammation (Yuan et al., 2012). Wnt5a is known to bind preferentially the atypical receptors Ror2 and Ryk; interestingly, both receptors have been reported to play a role in neuropathic pain models (Liu et al., 2015; Yang et al., 2017; Zhu et al., 2018; Zhou et al., 2019).

Neuronal plasticity is a key determinant of the transition from acute to chronic pain. Importantly, plasticity is not only functional in nature, but can also involve activity-dependent structural alterations, which hold tremendous potential for altering excitability in the long term. Here, we sought to address whether structural plasticity in spinal neurons in conditions of neuropathic and inflammatory pain involves Wnt signaling, and we explored the pathways involved using molecular, pharmacological, morphometric, and behavioral methods. We report that Wnt3a acting in the periphery leads to a release of Wnt5a in the spinal cord, which in turn spinally sustains nociceptive sensitization by increasing the density of dendritic spines in spinal neurons.

\section{Materials and Methods}

Mice and nociceptive tests. All animal procedures were performed in agreement with ethical guidelines provided by the local governing body (Regierungspräsidium). We followed ARRIVE (Animal Research: Reporting of In Vivo Experiments) guidelines.

Age-matched C57BL/6 mice of both sexes (between 8 and 14 weeks old) were used throughout all experiments. The central facility of Heidelberg University housed the mice in groups of two to four mice/ cage with constant room temperature and a $12 \mathrm{~h}$ light/dark cycle. All analyses were conducted by experimenters who were blinded to the identity of the study groups.

All behavioral measurements were performed in age-matched awake and unrestrained mice of both sexes. The mice were randomly assigned to treatment groups, and the investigator was blinded to the identity of the groups being tested in all behavioral tests. Behavioral testing was performed during the light (day) phase.

Mice were habituated to the experimental setups at least three times before the analysis and 30-60 min immediately before each experiment. Mechanical sensitivity was tested by using von Frey monofilaments of different forces (Ugo Basile) ranging from 0.008 to $1.0 \times \mathrm{g}$. We then calculated the frequency of paw withdrawal responses to five applications of each filament (Schweizerhof et al., 2009).

Mouse model of inflammatory pain. For induction of paw inflammation, a $20 \mu \mathrm{l}$ volume of Complete Freund's Adjuvant (CFA; catalog \#F5881, Sigma-Aldrich) was injected unilaterally under isoflurane anesthesia into the intraplantar surface of the hindpaw (Hartmann et al., 2004).

Mouse model of neuropathic pain. We used the spared nerve injury (SNI) model for mimicking trauma-induced neuropathic pain (Decosterd et al., 2002). Briefly, mice were anesthetized using 1.5-2.5\% isoflurane, and the sciatic nerve was exposed following an incision on the lateral surface of the thigh. The common peroneal and tibial branches were tightly legated and a $2-3 \mathrm{~mm}$ portion of the distal nerve stump was removed, whereas the sural nerve was left intact. Mice that received a sham surgery underwent the same surgical operation without injury to the nerves.

Mouse model of cancer pain. We used the LL2 cancer model as described previously (Stösser et al., 2010). We used a cancer cell line isogenic with C57BL/6 mice, the lung carcinoma cells (LL/2, LLc1; catalog \#90020104, European Collection of Cell Cultures). The cancer cells were grown in DMEM culture medium with $4.5 \mathrm{~g} / \mathrm{L}$ glucose, L-glutamine, without pyruvate supplemented with $10 \%$ fetal bovine serum, and $1 \%$ penicillin/streptomycin (all from Thermo Fisher Scientific). 
After harvesting the cells using trypsin (Thermo Fisher Scientific), the cells were resuspended in PBS and counted. A $20 \mu \mathrm{l}$ volume of PBS containing $7 \times 10^{5}$ cells was injected subcutaneously into the plantar surface of the hindpaw of isoflurane-anesthetized mice. To minimize suffering associated with tumor burden and cancer pain, the mice were killed before day 12 after tumor induction (Stösser et al., 2010).

Cell culture and treatments. After killing 6- to 8-week-old mice with $\mathrm{CO}_{2}$, the DRGs were quickly collected in cold PBS, and after removing axons and meningeal tissue they were digested for $30 \mathrm{~min}$ at $37^{\circ} \mathrm{C}$ in an enzyme mix containing trypsin, collagenase, and DNase. A discontinuous Percol gradient was used to separate neurons from non-neuronal cells. Purified neurons were plated on poly-L-lysine-coated coverslip and cultured in F12 medium plus 10\% normal serum, a mixture of growth factors (BDNF $10 \mathrm{ng} / \mathrm{ml}$, NGF $10 \mathrm{ng} / \mathrm{ml}$, GDNF $5 \mathrm{ng} / \mathrm{ml}$, and NT3 $5 \mathrm{ng} /$ $\mathrm{ml}$, all from Sigma-Aldrich), and the mitotic inhibitor AraC (5 $\mu \mathrm{M}$, Sigma-Aldrich) overnight before treatment.

The day after plating, the DRG neurons were treated with $10 \mathrm{ng} / \mathrm{ml}$ Wnt3a or vehicle for $3 \mathrm{~min}$. The cell culture was quickly collected, centrifuged at $4^{\circ} \mathrm{C}$ for $10 \mathrm{~min}$ and either frozen at $-80^{\circ} \mathrm{C}$ for subsequent analyses or immediately processed for Wnt5a-ELISA assay [Mouse Protein Wnt-5a (WNT5A) ELISA Kit, catalog \#CSB-EL026138MO, Cusabio] according to the manufacturer instructions.

Immunohistochemistry on mouse spinal cord. After perfusing mice transcardially with cold PBS, followed by $4 \%$ cold paraformaldehyde, the L3-L4 spinal cord segments were quickly extracted, cryopreserved in $30 \%$ sucrose, and sectioned at $20 \mu \mathrm{m}$ with a cryostat (Leica). Following $\mathrm{Na}$-Citrate antigen retrieval treatment, the sections were stained with the following antibodies: sheep anti-Ryk (1:25; catalog \#AF4649, R\&D Systems), rabbit anti-Ror2 (1:100; catalog \#PA5-77 028, Thermo Fisher Scientific), rabbit anti-PSD-95 (1:100; catalog \#51-6900, Thermo Fisher Scientific), mouse anti-PSD-95 (1:100; anti-PSD-95 MAGUK scaffold protein, NeuroMab clone K28/74, catalog \#75-348, UC Davis/NIH NeuroMab Facility; RRID:AB_2315909), mouse anti-Gephyrin (1:500; catalog \#147021, SySy), and rabbit anti-Gephyrin (1:1000; catalog $\# 147018$, SySy). After overnight treatment at $4^{\circ} \mathrm{C}$, the sections were washed and incubated with secondary antibodies for $1 \mathrm{~h}$ at room temperature. The following secondary antibodies were used: donkey antimouse Alexa Fluor (1:1000), donkey anti-rabbit Alexa Fluor (1:1000), and donkey anti sheep Alexa Fluor (1:1000), all from Thermo Fisher Scientific.

All the immunochemical experiments were performed by using standard protocols (Gangadharan et al., 2011) and were analyzed using a confocal laser-scanning microscope (model TCS SP2 AOBS or model TCS SP8 AOBS, Leica).

siRNA treatment. siRNAs against Ryk and Ror2 (Qiagen) were delivered in vivo using the in vivo-jetPEI reagent (Polyplus Transfection) according to the manufacturer directions. Briefly, we prepared the siRNA/polyethylenimine (PEI) complex by mixing solution A $(2 \mu \mathrm{g}$ of siRNA with $10 \%$ glucose solution, $1: 1)$ with solution B $(0.24 \mu \mathrm{l}$ of PEI solution with $10 \%$ glucose solution, 1:1) and incubated it for $15 \mathrm{~min}$ before use. Five microliters of solution was intrathecally injected once per day for 3 consecutive days.

Intrathecal injections. The injection method was described previously (Njoo et al., 2014). Briefly, animals were anesthetized with $2.5 \%$ isoflurane and intrathecal injection $(5 \mu \mathrm{l})$ was performed with a 30 gauge $\times 1 / 2$ needle attached to a $10 \mu$ l syringe (model 1701 LT SYR, catalog \#80,001, Hamilton). The needle tip was placed through the gap between lumbar vertebrae 5 and 6 . A successful intrathecal penetration was judged by the observation of tail twists.

RNA extraction and quantitative real-time PCR. Tissues from the L3-L5 DRGs or cultured DRGs were quickly collected and shock frozen on dry ice. After extracting total RNA using the TRIzol (Thermo Fisher Scientific), purification steps using Turbo DNase (Ambion), and RNaseOUT (Thermo Fisher Scientific) were used as per manufacturer instructions. The first-strand cDNA was retrotranscribed using $1 \mu \mathrm{g}$ of total RNA oligo(dT)20 primers (Thermo Fisher Scientific) and SuperScript III reverse transcriptase (Thermo Fisher Scientific), according to the manufacturer instructions. As a control, in some samples, reverse transcriptase was omitted.
Quantitative PCRs were run on cDNA samples using SyberGreen Gene Expression Assay (Roche) using specific primers for Ryk or Ror2, and GAPDH as the housekeeping gene (Sigma-Aldrich). The reactions were performed using a LightCycler 96Real-Time PCR System (Roche), and the data were analyzed using the corresponding software. The expression level of the target mRNA was normalized to the expression of Gapdh RNA. The quantitative real-time PCR (qRT-PCR) assay was performed in triplicate. The following primers were used: Ror2 sense, CCACAGCCATCGCCTATC; Ror2 antisense, GCAGACGAGGAAG AACAGG; Ryk sense, TGACAGCATCAGCGCCAGCA; Ryk antisense, CGCAAGTCGTTCTTCTCTATCCCGA; GAPDH sense, AGAAGG TGGTGAAGCAGGCATC; and GAPDH antisense CGAAGGTGGAA GAGTGGGAGTTG.

Golgi-Cox staining and tissue preparation. Golgi-Cox staining was performed using an FD Rapid GolgiStain Kit (FD Neurotechnologies), as described previously (Simonetti et al., 2013). Briefly, mice were killed just after behavioral testing or at corresponding time points as described in Results. Fresh, unfixed L3-L5 spinal cord segments were quickly removed, washed in distilled water, and treated with impregnation solution for $12 \mathrm{~d}$, followed by solution C for $2-5 \mathrm{~d}$ (FD Neurotechnologies) according to the manufacturer instructions.

The tissues were then frozen in dry ice, and sectioned on a cryostat at $150 \mu \mathrm{m}$ thickness and mounted on gelatinized glass slides. Sections were stained, dehydrated, cleared, and coverslipped according to the manufacturer instructions.

For analysis of dendritic spines, we selected neurons located within laminae II or V with at least one dendrite extended into an adjacent lamina (relative to the origin of the cell body) that were completely impregnated and had a cell body diameter $15-20 \mu \mathrm{m}$ (Tan et al., 2008; Simonetti et al., 2013). Images were captured with a Nikon Eclipse Ni-E upright automated microscope with a Nikon DS-Qi1Mc cooled highly sensitive CCD camera.

ELISA for determination of Wnt5a concentration. To detect whether Wnt5a was released at spinal level under different conditions in models of chronic pain, we performed an ELISA (Mouse Protein Wnt-5a (WNT5A) ELISA Kit, catalog \#CSB-EL026138MO, Cusabio) according to the manufacturer instructions. Briefly, ipsilateral and contralateral parts of the L3-L5 spinal laminae were collected separately after different treatments ( $1 \mathrm{~h}$ after intraplantar Wnt3a injection, $24 \mathrm{~h}$ after CFA injection, $6 \mathrm{~d}$ after LL2 cell injection or $3 \mathrm{~d}$ after SNI surgery) and processed as described by the manufacturer instructions.

After incubating with TMB (tetramethylbenzidine) substrate, the optical density was determined in each well using a microplate reader set to $450 \mathrm{~nm}$. A second reading was set at $540 \mathrm{~nm}$ for wavelength correction. The concentration of Wnt5a was determined by performing a standard curve according to manufacturer directions. In experiments involving spinal cord tissue, $2.5 \mathrm{mg}$ of tissue was used per sample, and Wnt5a content data were represented as pictograms per milligram of tissue.

To normalize the results of experiments involving DRG neurons in coculture to the protein input, the pellet obtained from the sample preparation was processed and Western immunoblotting analysis with an anti- $\beta$-tubulin III antibody (1:6000; catalog \#T2211, Sigma-Aldrich) was performed.

Statistics. All data are expressed as the mean \pm SEM. Statistically significant differences were analyzed using a Student's $t$ test to compare two groups with each other for a single parameter and a single time point. ANOVA for random measures or repeated measures (in longitudinal experiments) followed by post hoc Tukey's test for multiple comparisons was used to compare multiple groups or multiple time points. All the statistical tests are two sided unless otherwise specified in the figure legend. A $p$ value $\leq 0.05$ was considered significant. The $p$ values are provided in the figure legends.

\section{Results}

Wnt5a content is increased in the spinal dorsal horn in models of chronic pain

In our previous work, we found that in pathologic conditions, Wnt3a, released from cancer or inflammatory cells in the 


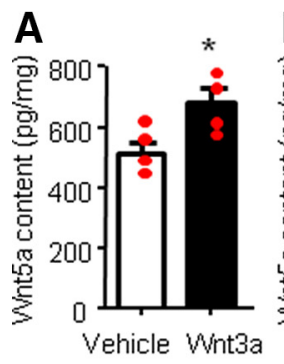

$\mathbf{F}$

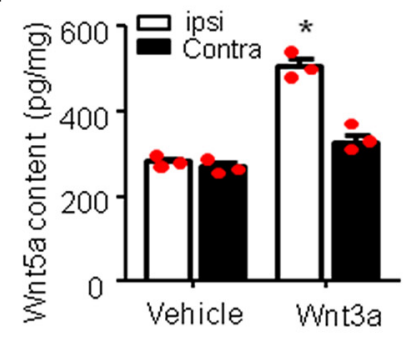

I

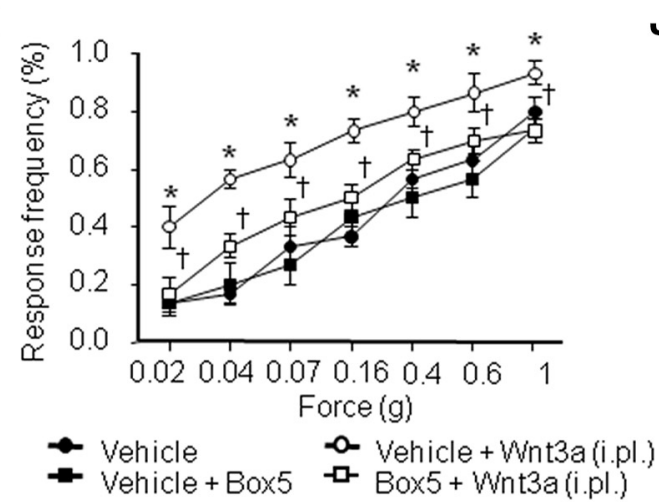

K

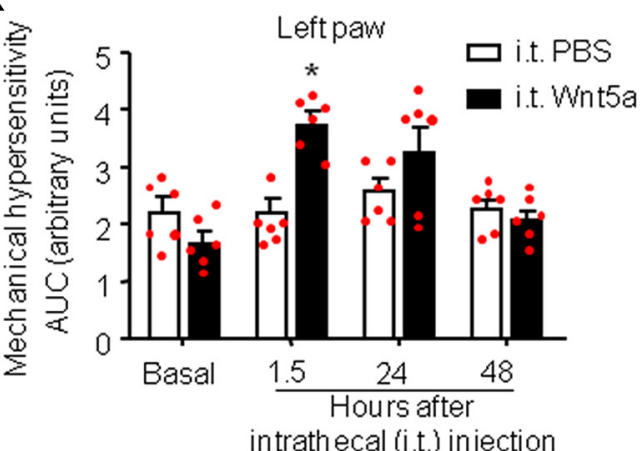

C

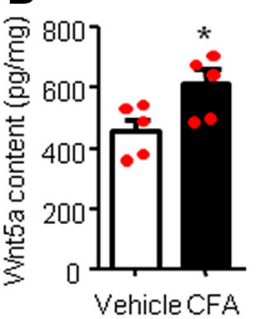

G

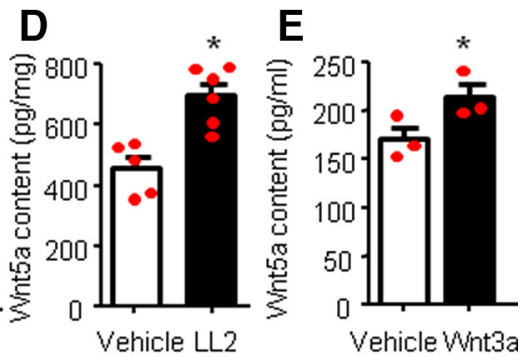

H
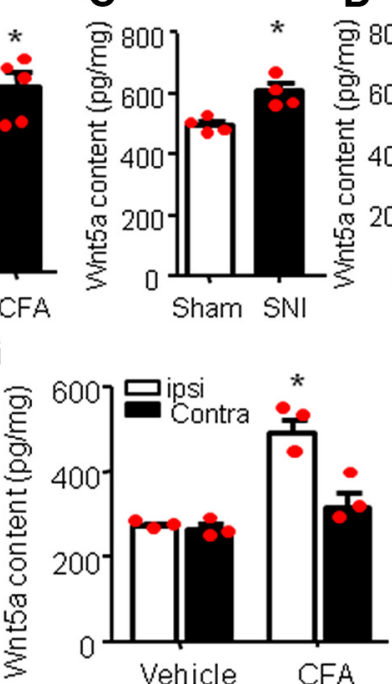
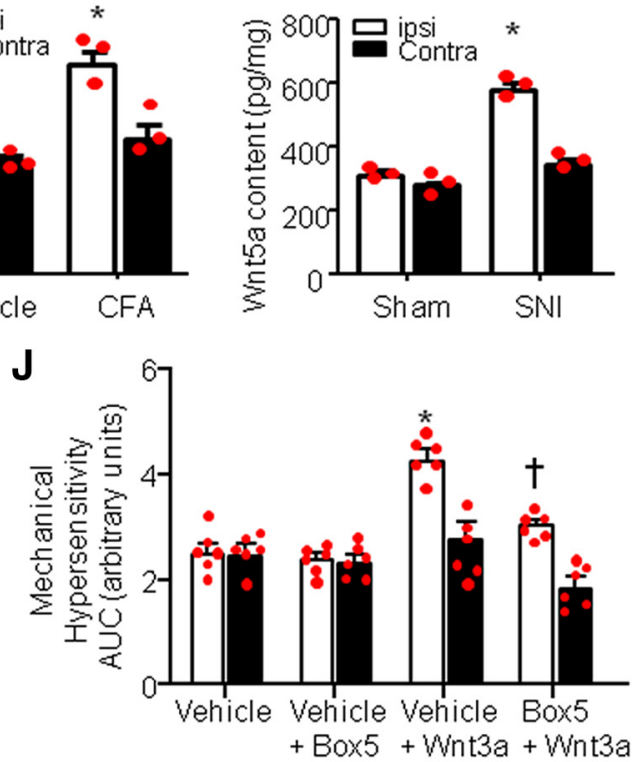

口 |psilateral

- Contralateral

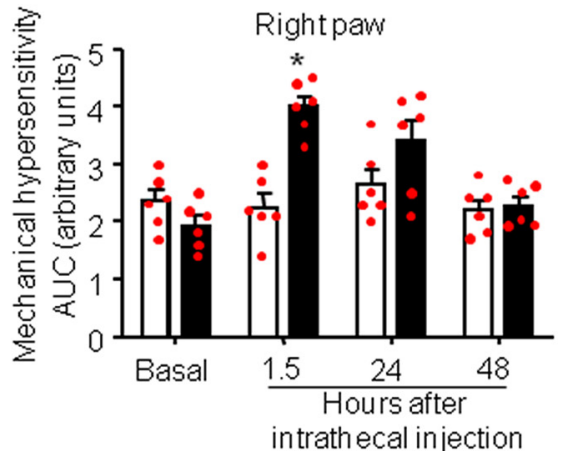

Figure 1. Wnt5a content is increased in the spinal dorsal horn in response to peripheral Wnt3a signaling, and in models of chronic pain and spinal Wnt5a mediates peripheral Wnt3ainduced nociceptive hypersensitivity. $\boldsymbol{A}-\boldsymbol{D}$, ELISA-based analysis of Wnt5a content bilaterally in spinal dorsal horns in vivo after intraplantar unilateral injection of recombinant mouse Wnt3a $(10 \mathrm{ng} / 20 \mu \mathrm{l} ; \boldsymbol{A})$, on inducing unilateral inflammation (intraplantar (FA injection; $\boldsymbol{B})$, after SNI $(\boldsymbol{C})$, or in a model of cancer pain (tumor growth induced by LL2 lung carcinoma cells in the calcaneus heel bone of the hindpaw; $\boldsymbol{D}) . N=3-4$ mice/group. Student's $t$ test was performed. ${ }^{*} p<0.05$ compared with vehicle treatment. $\boldsymbol{E}$, ELISA-based analysis of release of Wnt5a from DRG neurons in culture after treatment with recombinant Wnt3a $(10 \mathrm{ng} / \mathrm{ml}) . \mathrm{N}=3$ independent cultures/group. Student's $t$ test was performed. ${ }^{*} p<0.05$ compared with vehicle treatment. $\boldsymbol{F}-\boldsymbol{H}$, ELISA-based analysis of Wnt5a content in ipsilateral versus contralateral spinal dorsal horns in vivo levels after intraplantar unilateral injection of recombinant mouse Wnt3a (10 ng/20 $\mu$ l; $\boldsymbol{F}$ ), on unilateral (FA-induced paw inflammation $(\boldsymbol{G})$, or after unilateral SNI $(\boldsymbol{H}) . N=3$ mice/group. Student's $t$ test was performed. ${ }^{*} p<0.05$ compared with vehicle treatment. $\boldsymbol{I}, \boldsymbol{J}$, Behavioral analysis of the effects of intrathecal administration of Box $(2 \mu \mathrm{g} / \mu \mathrm{l})$, a specific inhibitor of Wnt5a signaling (applied $30 \mathrm{~min}$ before Wnt3a application), on mechanical sensitivity measured $1 \mathrm{~h}$ after intraplantar unilateral paw injection of Wnt3a $(10 \mathrm{ng} / 20 \mu \mathrm{l})$. Integral of response frequency-von Frey force intensity curves over time for the Wnt3a-injected (ipsilateral) paw and the contralateral paw are shown in $J . N=8$ mice/group; ANOVA for repeated measures was performed, followed by Tukey's test; ${ }^{*} p<0.05$ compared with the corresponding control group; $t p<0.05$ compared with Wnt3a-treated animals. $\boldsymbol{K}$, Effect of intrathecal injection of recombinant mouse Wnt5a on mechanical sensitivity of mouse hindpaw, shown as integral of response frequency-von Frey force intensity curves over time for both paws. $N=8$ mice/group. ANOVA for repeated measures followed by Tukey's test was performed. ${ }^{*} p<0.05$ compared with the corresponding control groups. In all panels, data are represented as the mean \pm SEM, and bar graphs are also shown as scatter plots. ipsi, Ipsilateral; Contra, contralateral; i.pl., intraplantar; i.t., intrathecal. 
periphery, was able to induce sensitization of primary afferents via activation of canonical and noncanonical signaling pathways (Simonetti et al., 2014). Furthermore, Wnt3a was shown to be released from hippocampal synapses in an activity-dependent and NMDA receptor-mediated manner (Chen et al., 2006). Interestingly, mouse DRG neurons express the noncanonical ligand Wnt5a but not the canonical ligand Wnt3a (Simonetti et al., 2014). Since we found that Wnt3a application on cultured DRG neurons was able to induce the release of inflammatory cytokines such as IL- $1 \beta$ or TNF- $\alpha$ (Simonetti et al., 2014), we asked whether hindpaw injection of Wnt3a or painful stimuli would induce enhancement of the Wnt5a content in the spinal dorsal horn.

Performing an ELISA, we found that peripheral application of Wnt3a via unilateral paw injection was able to increase the spinal Wnt5a content (Fig. 1A), compared with vehicleinjected mice (increment of $36.66 \pm$ $2.2 \%, p<0.05, n=4)$. Importantly, performing an ELISA on spinal samples from mouse models of inflammatory, cancer, or neuropathic pain, we found increased spinal cord Wnt5a content across all of these chronic pain conditions compared with vehicle treatment or sham operation (increment of $25 \pm 1.4 \%$ for the CFAinduced inflammation; $p<0.05, n=5$ vehicle treatment, $n=4 \mathrm{CFA}$ inflammatory model, Fig. $1 B$; increase of $20 \pm 8.3 \%$ for SNI-induced neuropathic pain model; $p<0.05, n=4$ sham operated, $n=4$ SNI, Fig. $1 C$ ) and an increase of $40 \pm 4.8 \%$ for the LL2mediated cancer model $[p<0.05$, $n=5$ control (injection of dead [boiled] LL2 cells), $n=6$ cancer model, Fig. $1 D]$. To address whether this significant, but modest, increase in Wnt5a could have arisen due to dilution across ipsilateral and contralateral dorsal horns, we performed a separate series of experiments to directly compare ipsilateral and contralateral changes in Wnt5a content. Only ipsilateral, not contralateral, changes were significant, and we observed that Wnt5a content is increased ipsilaterally in the spinal cord by $55 \pm 5.2 \%$ over vehicle following Wnt3a injection in the paw (Fig. $1 F$ ), by $52.3 \pm 4.7 \%$ over sham in mice with CFA-induced unilateral paw inflammation (Fig. 1G), and by $66.2 \pm 3.8 \%$ over sham in mice with SNI (Fig. $1 H$ ).

Because we have previously shown that these chronic pain models elicit Wnt3a in the periphery, followed by nociceptor sensitization by Wnt3a, our results of Wnt3a-induced Wnt5a in

A

C

D
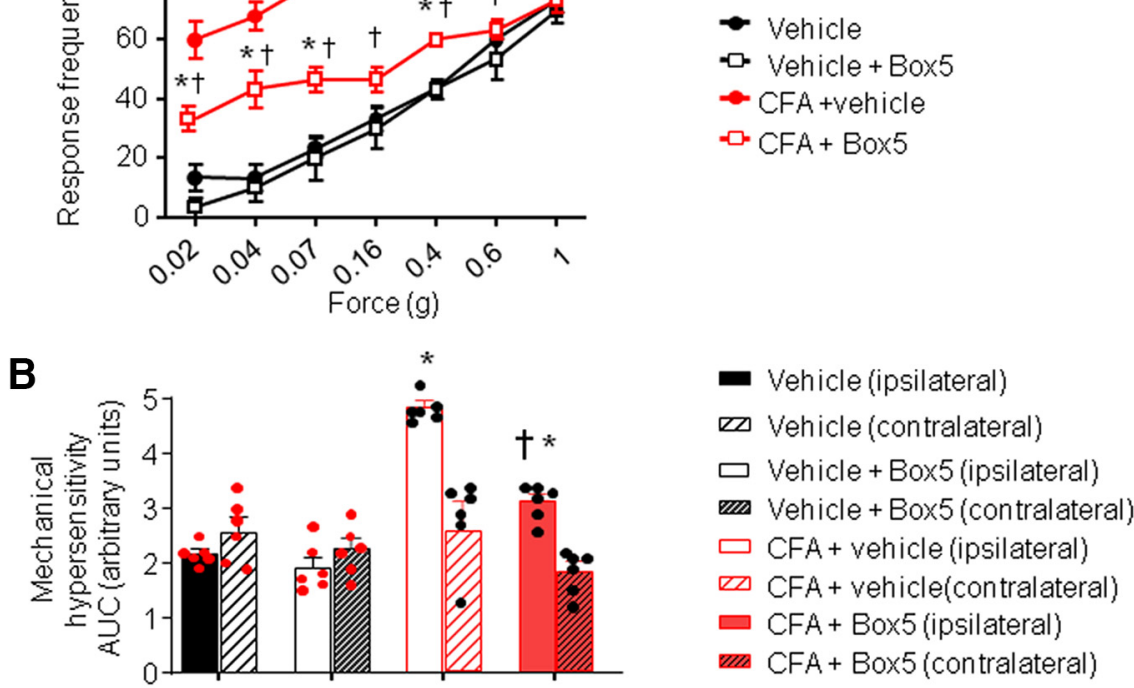

Figure 2. Increase in spinal Wnt5a content contributes to nociceptive hypersensitivity in inflammatory and neuropathic models. A-D, Behavioral analysis of the effects of intrathecal (i.t.) administration of Box5 $(2 \mathrm{ng} / \mu \mathrm{ll})$, a specific inhibitor of Wnt5a signaling, on mechanical sensitivity measured $24 \mathrm{~h}$ after (FA-induced unilateral paw inflammation $(\boldsymbol{A}, \boldsymbol{B})$ or $3 \mathrm{~d}$ after unilateral nerve injury $(\mathrm{SNl} ; \boldsymbol{C}, \boldsymbol{D})$. Box5 was applied $30 \mathrm{~min}$ before mechanical behavioral testing. Stimulus-response curves of the ipsilateral paw are shown in $\boldsymbol{A}$ and $\boldsymbol{C}$, whereas their integral over time (AUC) for the inflamed or injured paw (ipisilateral) and the contralateral paw are shown in $\boldsymbol{B}$ and $\boldsymbol{D}$. In all panels, $n=8$ mice/group; ANOVA for repeated measures was performed, followed by Tukey's test; ${ }^{*} p<0.05$ compared with the corresponding control group; $\uparrow p<0.05$ compared with mice with (FA (A) or SNI (C). In all panels, data are represented as the mean \pm SEM, and bar graphs are also shown as scatter plots.

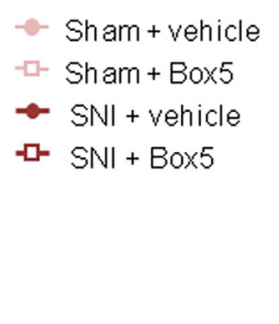

\footnotetext{
Sham + vehicle (ipsilateral) Sham + vehicle (contralateral) Sham + Box5 (ipsilateral) Sham + Box5 (contralateral) - SNI + vehicle (ipsilateral)

z S SNI + vehicle (contralateral)

$\square$ SNI + Box5 (ipsilateral)

D SNI + Box5 (contralateral)
} 
A
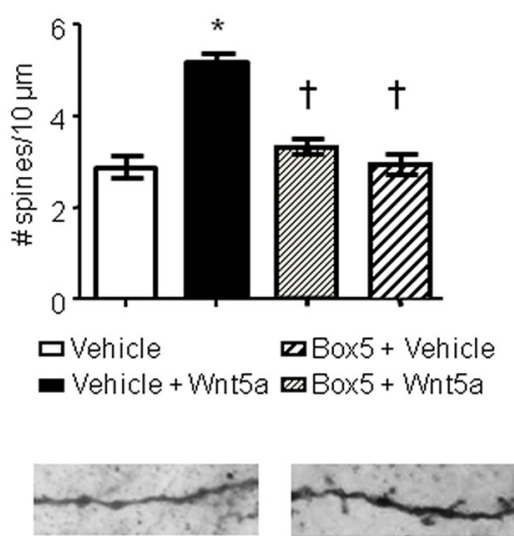

Vehicle

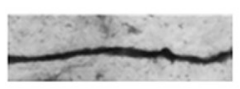

Bo $\times 5+$ Vehicle

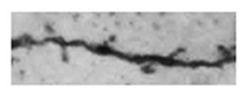

Vehicle + Wnt5a

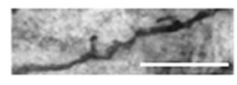

Box5+ Wnt5a
B
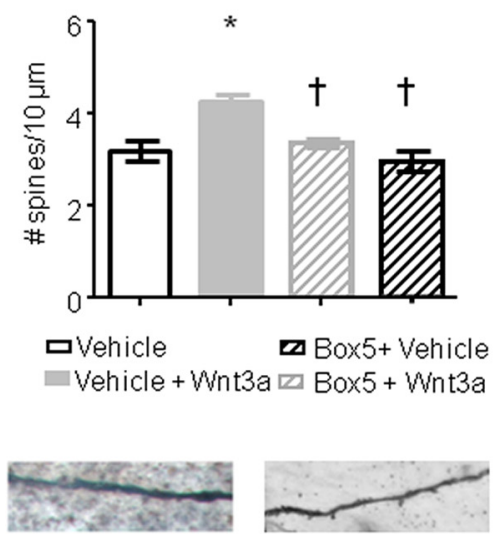

Vehicle

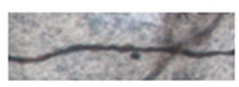

Box5 + Vehicle

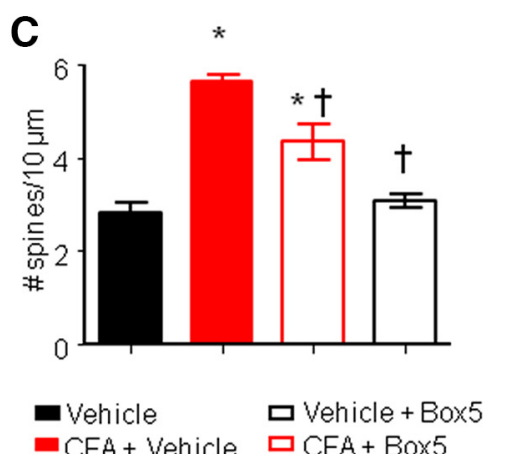

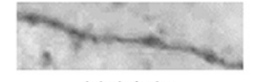

Vehicle

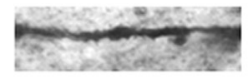

Vehicle + Box 5

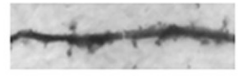

CFA + Vehicle

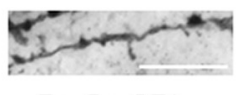

Figure 3. Spinal Wnt5a induces remodeling of synaptic spines in spinal dorsal horn neurons. A-D, Quantification of synaptic spine density in spinal neurons (primarily of lamina V origin) in vivo following intrathecal injection of Wnt5a $(2 \mu \mathrm{g} / 5 \mu \mathrm{l} ; A)$, intraplantar injection of Wnt3a $(10 \mathrm{ng} / 20 \mu \mathrm{l} ; \boldsymbol{B}), 24 \mathrm{~h}$ after CFA injection (C) or $7 \mathrm{~d}$ after nerve lesion (D), in the presence or absence of intrathecal administration of Box5, a Wnt5a signaling inhibitor. Examples of high-magnification views of microscopic images of labeled dendrites with synaptic spines are shown below the corresponding bar graphs. Scale bar, $10 \mu \mathrm{m} ; \mathrm{N}=3-4$ animals/condition, 20-30 neurons, 500-700 spines counted were analyzed. Two-way ANOVA for random measurements, followed by Bonferroni's test, was performed. ${ }^{*} p<0.05$ compared with control group (vehicle or sham treated); $\uparrow p<0.05$ compared with the group that received only the nociceptive stimuli but not the inhibitor. In all panels, data are represented as the mean \pm SEM.

increase of Wnt5a content compared with vehicle-treated cultures $(p<0.05, n=3$ for each group; Fig. $1 E)$. Thus, we conclude that Wnt5a content increases in the spinal cord in an activity-dependent manner following stimulation of DRG neurons by chemicals or by pain mediators and that Wnt3ainduced sensitization of DRG neurons can enhance Wnt5a content.

Role of spinal Wnt5a in mechanical hypersensitivity induced by peripheral Wnt3a

Since we found an increased Wnt5a content in the spinal cord following hindpaw injection of Wnt3a (Fig. 1A,E), we asked

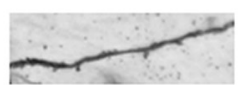

Vehicle + Wht3a

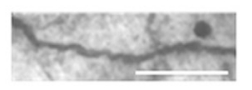

Box5 + Wht $3 a$ whether spinal Wnt5a could mediate Wnt3a-induced hypersensitivity. Therefore, $15 \mathrm{~min}$ before peripheral injection of Wnt3a, we intrathecally applied Box5, a specific blocker, which has been used in previous studies in pain models (10 ng/5 $\mu \mathrm{l}$; Yuan et al., 2012, 2015; Wang et al., 2015; Zhu et al., 2018) and is believed to inhibit Wnt5a signaling at receptors rather than sequestering Wnt5a (Jenei et al., 2009). After 1 h, we measured responses to mechanical von Frey stimuli application. Box 5 treatment was able to attenuate peripheral Wnt3a-induced mechanical hypersensitivity $(p<0.05$; Fig. $1 I$ ) without changing nociceptive sensitivity under normal conditions. When stimulus intensity-response frequency analyses were compared for all von Frey forces tested by calculating the area under the curve (AUC), we observed a significant decrease in the extent of Wnt3a-induced mechanical hypersensitivity in Box5-treated mice compared with the vehicle-treated mice (Fig. 1J). Interestingly, treatment with either Wnt3a or Box5 affects the mechanical sensitivity of the contralateral paw (Fig. 1J).

In support of these data, we found that direct intrathecal injection of recombinant Wnt5a $(20 \mathrm{ng} / 5 \mu \mathrm{l}$; Yuan et al., 2015) in wild-type mice was able to induce marked and rapid mechanical sensitization to von Frey stimuli with a time course resembling that of peripheral Wnt3a-induced mechanical hypersensitivity (see above). Indeed, intrathecal Wnt5a-induced sensitization arose within $30 \mathrm{~min}$, reached the peak by $1 \mathrm{~h}$, and disappeared within $24 \mathrm{~h}$ after a single application (Fig. $1 K$ ).

\section{Spinal increase in Wnt5a levels contributes functionally to mechanical hypersensitivity in chronic pain conditions}

Since Wnt5a increases in spinal cord in chronic pain conditions (Fig. $1 B-D$ ), we addressed whether spinal blockade of Wnt5a was able to reduce neuropathic and/or inflammatory pain in the SNI and CFA mouse models, respectively. Box5 treatment, 24 $\mathrm{h}$ after CFA injection, was able to attenuate at least partially inflammatory pain (Fig. 2A). We observed a significant decrease in the extent of CFA-induced mechanical hypersensitivity $24 \mathrm{~h}$ after CFA injection in Box5-treated mice compared with the vehicle-treated mice (Fig. $2 B$ ). Box5 treatment was also partially blocked SNI-induced mechanical hypersensitivity, when injected $7 \mathrm{~d}$ after surgery compared with the vehicletreated SNI group (Fig. 2C,D). Interestingly, Box5 treatment does not affect the mechanical sensitivity of the contralateral paw after either inflammation or nerve injury (Fig. 2B,D). 
Thus, we conclude that blocking spinal Wnt5a at least partially contributes to plasticity in inflammatory and neuropathic pain.

\section{Wnt5a induces structural remodeling of synaptic spines on spinal dorsal horn neurons}

Recently, work from our laboratory and others have demonstrated a key role for dendritic spine plasticity in the dorsal horn of spinal cord in inducing and maintaining nociceptive hypersensitivity (Tan et al., 2011; Simonetti et al., 2013). Developmentally, Wnt5a has been implicated in synapse formation, differentiation, and function (Varela-Nallar et al., 2010); we asked whether Wnt5a-induced mechanical hypersensitivity could be mediated by the altering dendritic spines in the dorsal horn.

To assess changes in neuronal morphology, we performed Golgi staining on spinal tissue of mice treated intrathecally with either Wnt5a or vehicle. We measured mechanical sensitivity to von Frey filament application $1 \mathrm{~h}$ after intrathecal injection and immediately terminated the experiment to obtain tissue for spine analysis. Neurons in the most superficial lamina (i.e., lamina I) are largely aspiny, while neurons in lamina II and mostly the large pyramidal neurons in lamina $\mathrm{V}$ neurons show spines (Tappe et al., 2006). In our hands, mostly lamina $V$ neurons and a few lamina II neurons were impregnated by Golgi staining and were subjected to spine analyses. Quantifying spine-like dendritic protrusions in Golgi-stained dorsal horn neurons revealed that spine density was significantly increased in Wnt5atreated mice over vehicle-treated mice $(5 \pm 0.38 / 10 \mu \mathrm{m}$ vs $3 \pm 0.28 ; p<0.05$; $n=3$ different mice; Fig. $3 A$ ).

Interestingly, peripheral injection of Wnt3a was also able to increase spine density in the dorsal horn albeit with a lower magnitude $(4.3 \pm 0.3 /$ $10 \mu \mathrm{m}$; Fig. 3B). To understand whether this effect was directly mediated by Wnt5a, we analyzed Golgi-stained spinal cord from Box5-pretreated mice. We found that the intrathecal treatment with Wnt5a blocker was able to completely abrogate the effects of both intrathecal Wnt5a or peripheral Wnt3a treatments on dorsal horn spine density (Fig. $3 A, B$ ). The Wnt ligand-induced increase in spine density detected in mice pretreated with Box 5 was significantly lower than the magnitude seen in mice treated either with Wnt5a or Wnt3a and was comparable to the spine density detected in vehicle-treated mice $(3.4 \pm 0.3 / 10 \mu \mathrm{m})$.
A

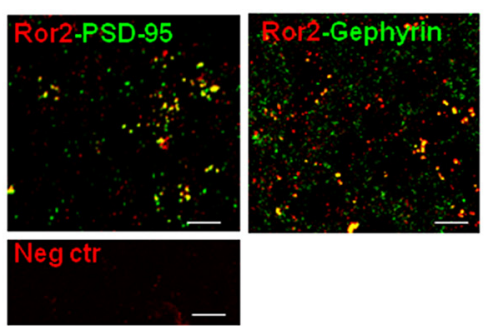

B
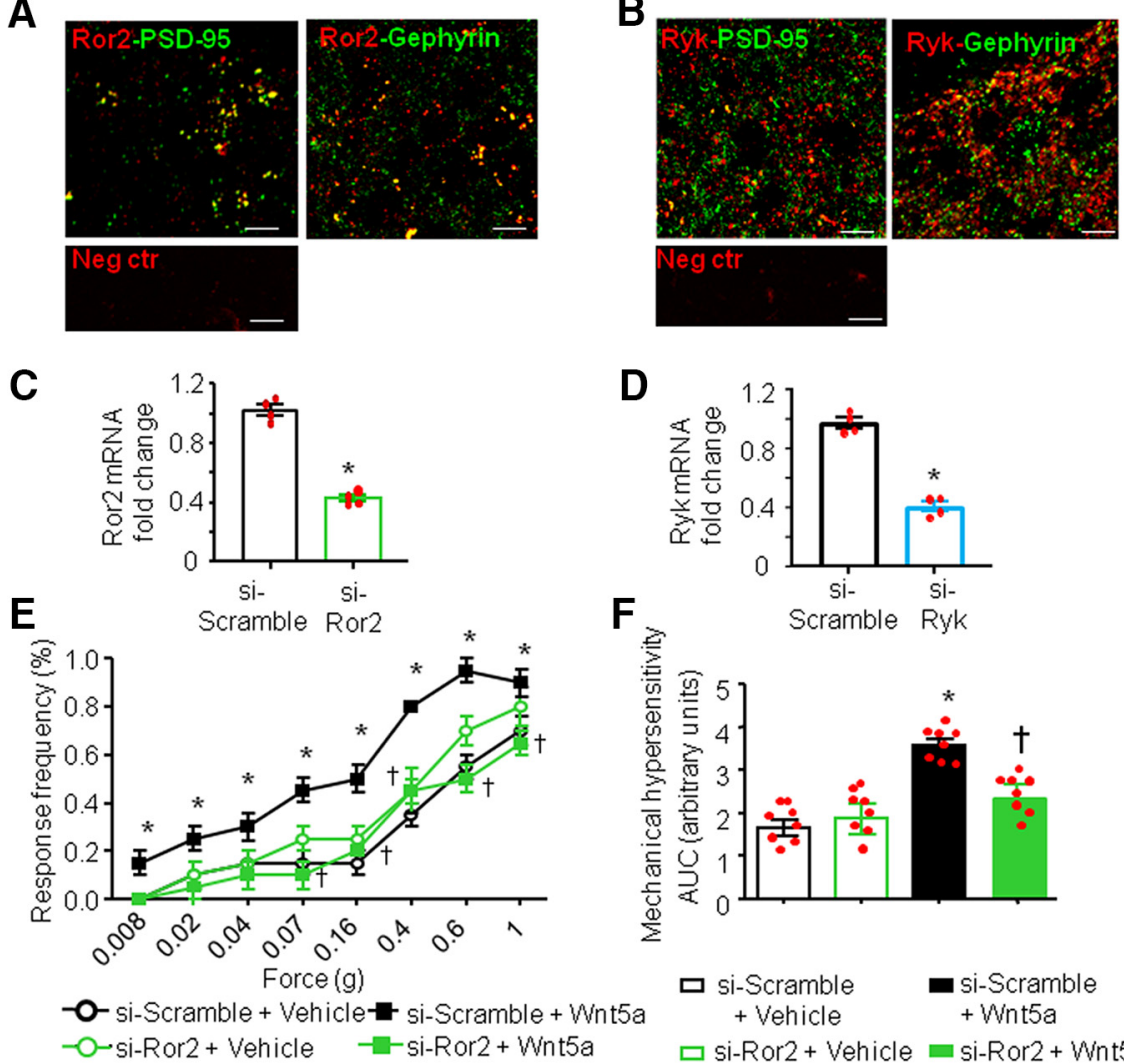

D
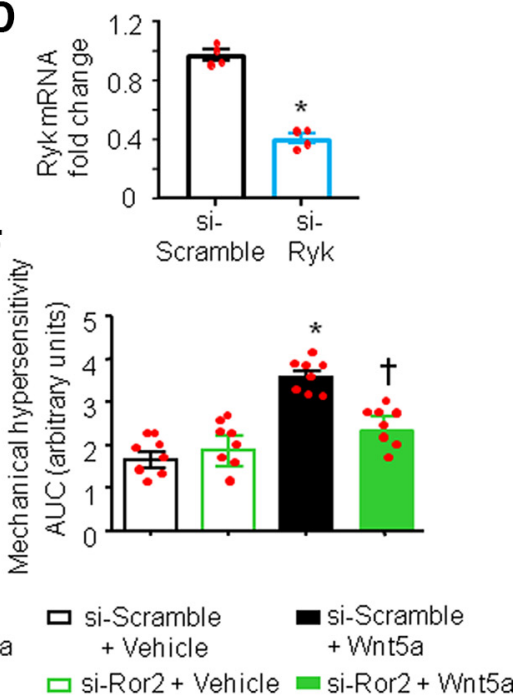

G

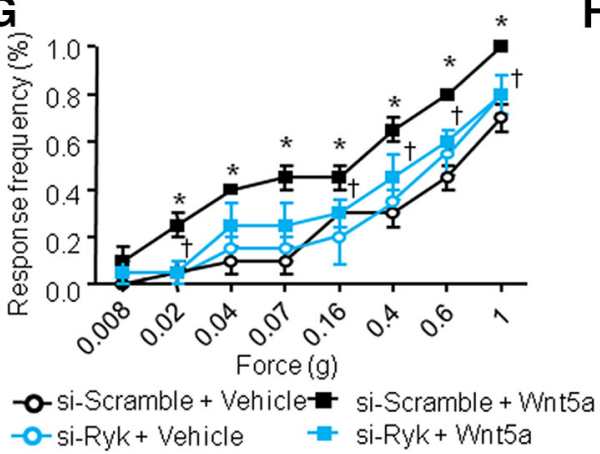

$\mathrm{H}$

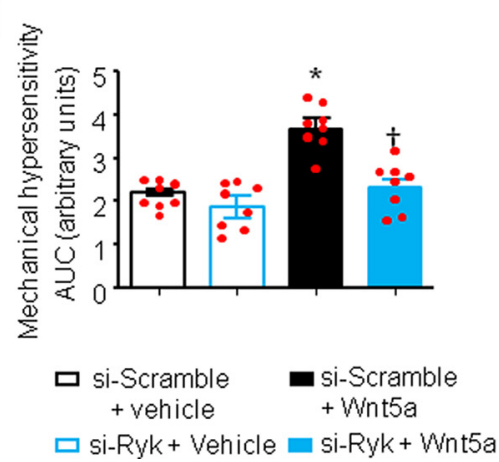

Figure 4. Noncanonical Ror2 and Ryk receptors mediate spinal Wnt5a-induced nociceptive hypersensitivity. $\boldsymbol{A}, \boldsymbol{B}$, Typical example of coimmunoreactivity for Ror2 $(\boldsymbol{A})$ or Ryk ( $\boldsymbol{B}$; both in red) in the spinal dorsal horn, together with the markers for excitatory synapses (PSD-95, left) or inhibitory synapses (Gephyrin, right; both in green). Bottom panels show negative controls. Scale bar, $10 \mu \mathrm{m}$. C, D, Evidence for marked RNA interference-mediated knockdown of Ror2 (C) or Ryk (D) mRNA in the spinal dorsal horn on intrathecal injection of siRNA against Ror2 (si-Ror2) or Ryk (si-Ryk), respectively, in relation to intrathecal injection of the respective scrambled siRNAs as controls (si-Scramble). $N=4$ mice/condition. Student's $t$ test was performed. ${ }^{*} p<0.05$ compared with the corresponding control group. $\boldsymbol{E}, \boldsymbol{H}$, Complete blockade of Wnt5a-induced mechanical hypersensitivity in mice intrathecally injected with siRNA against Ror2 $(\boldsymbol{E}, \boldsymbol{F})$ or siRNA against RyK $(\boldsymbol{G}, \boldsymbol{H})$ compared with mice injected with scrambled siRNA ( $n=6-7$ mice/group). Stimulus-response curves of the one paw are shown in $\boldsymbol{E}$ and $\boldsymbol{G}$, whereas their integral over time (AUC) are shown in $\boldsymbol{F}$ and $\boldsymbol{H}$. Two-way ANOVA followed by Tukey's test was performed. ${ }^{*} p<0.05$ compared with the corresponding control group; $t p<0.05$ compared with si-Scramble + Wnt5a-treated animals. In all panels, data are represented as the mean \pm SEM, and bar graphs are also shown as scatter plots.

Recently, we and others (Simonetti et al., 2013; Tan and Waxman, 2015) have shown that both CFA-induced inflammation and SNI-induced nerve injury lead to dendritic spines rearrangement into the spinal dorsal horn with an increased number of dendritic spines and mediating at least partially mechanical hyperalgesia. Since we saw that blocking Wnt5a signaling in the spinal cord reduces CFA- or SNI-induced mechanical hyperalgesia, we tested whether Wnt5a could be operational in the spine remodeling occurring in these pathologic conditions. 
A

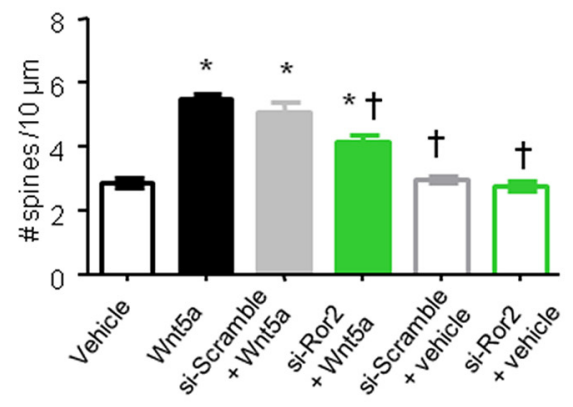

B

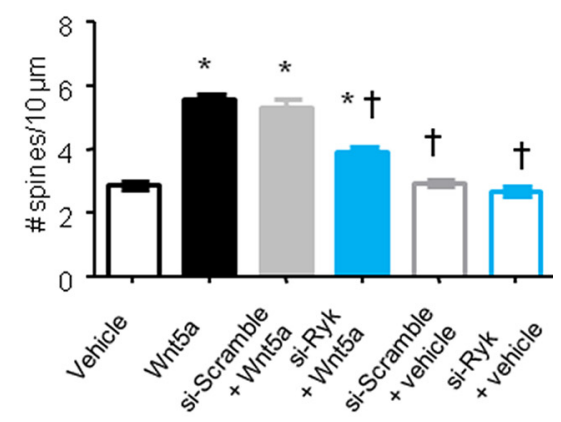

C

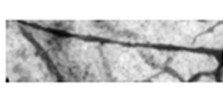

Control

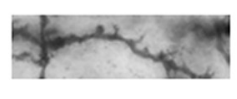

Wnt5a

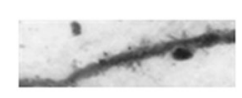

si-Scramble + Wnt5a
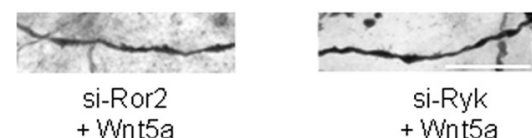

Figure 5. Ror2 and Ryk mediate spinal Wnt5a-induced dendritic spine remodeling. $\boldsymbol{A}, \boldsymbol{B}$, Quantification of synaptic spine density in spinal dorsal horn neurons of primarily lamina $V$ origin in mice intrathecally injected with recombinant mouse Wnt5a and receiving siRNA against Ror2 $(\boldsymbol{A})$, siRNA against Ryk $(\boldsymbol{B})$, or the respective scrambled siRNA controls $(\boldsymbol{A}, \boldsymbol{B})$. $N=3-4$ mice/condition. Two-way ANOVA for random measures, followed by Bonferroni's test, was performed. ${ }^{*} p<0.05$ compared with the corresponding control group; $t p<0.05$ compared with Wnt5a-treated mice. In all panels, data are represented as the mean \pm SEM. C, Typical examples of high-magnification views of microscopic images of Golgi-labeled dendrites with synaptic spines in mice from the above groups. Scale bar, $10 \mu \mathrm{m}$.

We found that the intrathecal Box 5 treatment $24 \mathrm{~h}$ after CFA injection or $7 \mathrm{~d}$ after SNI surgery significantly attenuated the effects of CFA-induced inflammation or SNI-induced nerve injury on dorsal horn spine density (Fig. 3C,D). CFA- or SNIinduced increase in spine density detected in Box5-treated inflamed or neuropathic mice was significantly lower compared with that seen in vehicle-treated inflamed mice $(4.2 \pm 0.3$ vs $5.8 \pm 0.2 / 10 \mu \mathrm{m}$, respectively; Fig. $3 C$ ) or vehicle-treated neuropathic mice ( $4.1 \pm 0.18$ vs $5.1 \pm 0.25 / 10 \mu \mathrm{m}$, respectively; Fig. $3 D$ ).

The noncanonical receptors Ror2 and Ryk mediate Wnt5ainduced structural plasticity and nociceptive hypersensitivity Wnt5a is known to bind the transmembrane receptor-like tyrosine kinases Ror2 and Ryk in addition to Frizzled receptors (Kikuchi et al., 2012). Both of these receptors were shown to bind directly to Wnt5a or to function as coreceptors participating in the formation of ternary complexes together with Wnt5a and Frizzleds (Yamamoto et al., 2008; Nishita et al., 2010). Since we found that both Ror2 and Ryk receptors were able to mediate Wnt5a-induced mechanical hypersensitivity, consistent with previous findings (Shi et al., 2012; Yuan et al., 2012; Liu et al., 2015; Yang et al., 2017; Zhu et al., 2018), and are linked to morphologic effects of Wnt signaling, we tested their role in Wnt5a-induced spine remodeling.

We first performed immunofluorescence analysis of the expression of both Ror2 and Ryk in the spinal cord dorsal horn of adult mice. Interestingly, the localization pattern of both Ror2 and Ryk resembled that of synaptic molecules, such as synapsin or PSD-95. Figure 4 shows a certain degree of colocalization with postsynaptic markers, Gephyrin for inhibitory synapses and PSD-95 for excitatory synapses for both Ror2 and Ryk (Fig. $4 A, B$ ).
To address the involvement of these two receptors in the modulation of pain sensitivity, we intrathecally injected specific siRNAs against Ror2 or Ryk (siRNA-Ror2 and siRNA-Ryk respectively). The efficacy of siRNA treatment in knocking down the expression of Ror2 and Ryk in the spinal dorsal horn in vivo was confirmed via $\mathrm{qRT}-\mathrm{PCR}$ analysis $24 \mathrm{~h}$ after the last siRNA injection (Fig. 4C,D). In both cases, we observed a reduction $>50 \%$ in the content of receptor-specific mRNA. In particular, the Ror2-siRNA treatment led to a $58 \pm 2.1 \%$ reduction of Ror 2 mRNA in the spinal cord, whereas the RyksiRNA treatment led to a $62.3 \pm 2.8 \%$ reduction of Ryk mRNA content in the spinal cord. In mice with intrathecal siRNA-Ror2 treatment, we observed a complete lack of Wnt5a-induced mechanical hypersensitivity (Fig. 4E,F; $p<0.05$ ) without any change in basal mechanical sensitivity. Surprisingly, we found that siRNA-Ryk treatment was also able to completely block Wnt5ainduced hypersensitivity (Fig. 4F,G), suggesting that both Ror2 and Ryk are needed in spinal neurons for Wnt5a signaling.

To investigate the role of Wnt5a/Ror2 or Wnt5a/Ryk pathways as possible modulators of synaptic spine density in vivo, immediately after the behavioral analyses mentioned above, the experiment was terminated and the spinal tissue processed quickly for Golgi staining.

The capacity of Wnt5a to increase spine density in spinal dorsal horn neurons was partially lost in siRNA-Ror2-treated mice, but not in mice expressing the siRNA-scramble control (5.7 \pm 0.3 vs $4.15 \pm 0.2$ spines $/ 10 \mu \mathrm{m} ; p<0.05$; Fig. $5 A$ ). Furthermore, consistent with the lack of impact on basal nociceptive sensitivity, siRNA-Ror2 treatment had no effect on dendritic spine density when applied under basal conditions in the absence of Wnt5a (i.e., in vehicle-treated mice; Fig. 5A). Pretreatment with siRNA-Ryk partially prevented the Wnt5a-induced enhancement of dendritic spines on spinal neurons ( $4 \pm 0.1$ spines for siRNARyk vs $5.8 \pm 0.2$ spines for siRNA-scramble $/ 10 \mu \mathrm{m} ; p<0.05$; Fig. 5B).

Together, these experiments provide evidence for a role for both Ror2 and Ryk as mediators of Wnt5a-induced mechanical hyperalgesia and dendritic spine plasticity.

\section{Spinal Ror2 and Ryk contribute to mechanical hypersensitivity in mouse models of inflammatory or neuropathic pain}

We next investigated the role of Ror2 and Ryk receptors as possible modulators of synaptic spine density in vivo in models of pathologic pain.

We found that intrathecal treatment with siRNA-Ror2 administrated before intraplantar CFA injection was able to attenuate CFA-induced mechanical hypersensitivity at $24 \mathrm{~h}$ after CFA injection compared with mice injected intrathecally with siRNA-scramble control (Fig. 6A,B). Indeed, mice lacking Ror2 
developed inflammatory mechanical hypersensitivity to a significantly lower extent than control mice (Fig. $6 A, B)$. There was no effect on sensitivity of the contralateral paw (Fig. 6B).

Given the limited time span of knockdown after siRNA injections, we knocked down Ror2 spinally in the SNI model of neuropathic pain $3 \mathrm{~d}$ postsurgery, when mechanical hyperalgesia is already evident. Compared with SNI mice receiving siRNA-scramble control, Ror2 knockdown significantly reduced mechanical nociceptive hypersensitivity when it is established at its peak at $7 \mathrm{~d}$ post-SNI (Fig. 6C,D). Contralateral sensitivity is shown in Figure $6 D$. These results indicate that both induction and maintenance of pathologic hypersensitivity can be diminished by Ror2 knockdown.

Similarly, on testing the impact of Ryk knockdown with similar protocols and timeframe, we observed that intrathecal treatment with siRNARyk also significantly attenuated inflammatory hypersensitivity as well as neuropathic mechanical hypersensitivity compared with siRNAscramble control (Fig. 7A,D); however, the magnitude of inhibition was lower than that seen with Ror2 knockdown. Contralateral sensitivity is shown in Figure 7, $B$ and $D$.

\section{Role of Ror2 and Ryk in spine plasticity in chronic pain models} We first induced Ror2 or Ryk knockdown at spinal cord level via intrathecal injection of specific siRNA, then we gave an intraplantar injection of CFA on day 4 to induce inflammatory pain. Mice injected intrathecally with either siRNA-Ror2 or siRNA-Ryk showed a significant decrease in spine density on spinal neurons compared with siRNAscramble-injected mice $(6 \pm 0.1$ for siRNA-scramble vs $4.3 \pm$ 0.15 for siRNA-Ror 2 spines/ $10 \mu \mathrm{m}$ vs $4.2 \pm 0.2$ for siRNA-Ryk spines $/ 10 \mu \mathrm{m} ; p<0.05$; Fig. $8 A, B$ ), suggesting that both of the receptors are involved in the synaptic plasticity that arises at the spinal level in inflammatory pain conditions.

Second, we used the SNI model of neuropathic model to test whether these two receptors were involved in the maintenance of neuropathy-induced spine morphogenesis. Three days after inducing nerve injury, when neuropathic pain is already well established, we knocked down either Ror2 or Ryk. These treatments were able to partially revert the increase of spine density at spinal level because of nerve injury (number of spines per $10 \mu \mathrm{m}$ : $5.2 \pm 0.1$ for siRNA-scramble vs $4.1 \pm 0.2$ for siRNA-Ror2 vs $4.0 \pm 0.1$ for siRNA-Ryk; $p<0.05$; Fig. $8 C, D$ ). This suggests that
A
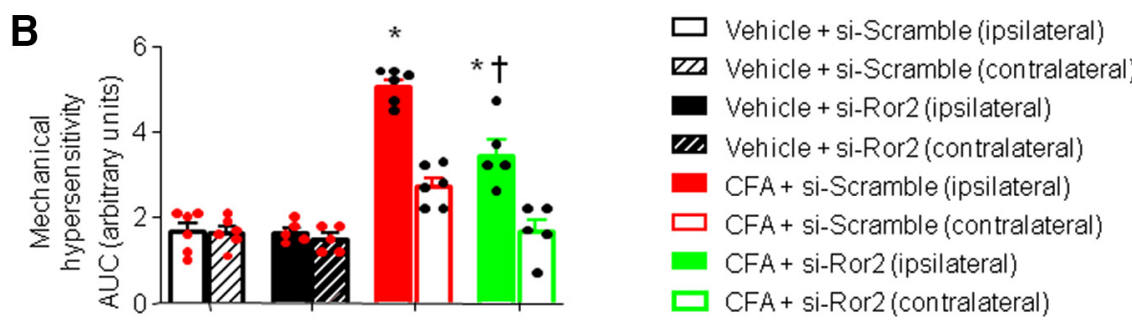

C

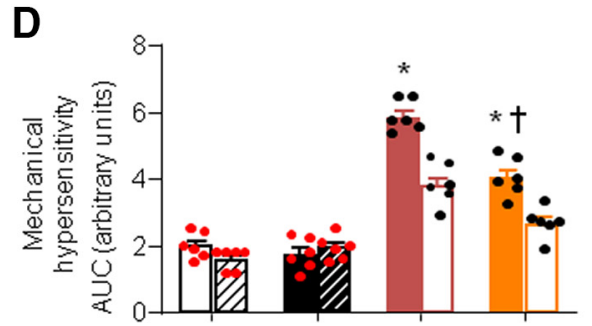

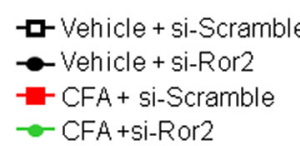

$\rightarrow$ CFA +si-Ror2

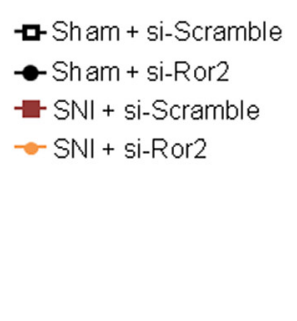

[7 Sham + si-Scramble (contralateral)

- Sham + si-Ror2 (ipsilateral)

II] Sham + si-Ror2 (contralateral)

SNI + si-Scramble (ipsilateral)

$\square$ SNI + si-Scramble (contralateral)

- SNI + si-Ror2 (ipsilateral)

SNI + si-Ror2 (contralateral)

Figure 6. Spinal Ror2 contribute to nociceptive hypersensitivity in mouse models of chronic pain. $\boldsymbol{A}-\boldsymbol{D}$, Behavioral analysis of the effects of intrathecal administration of siRNA against Ror2 on mechanical sensitivity $24 \mathrm{~h}$ after intraplantar injection of CFA-induced are shown in $\boldsymbol{A}$ and $\boldsymbol{C}$, whereas the integral over time (AUC) for the inflamed or injured paw (ipsilateral) as well as the contralateral paw are shown in $\boldsymbol{B}$ and $\boldsymbol{D}$. In all panels, $N=8$ mice/group; ANOVA for repeated measures was performed, followed by Tukey's test; ${ }^{*} p<0.05$ compared with the corresponding control group; $\uparrow p<0.05$ compared with CFA-treated $(\boldsymbol{A}, \boldsymbol{B})$ or SNI-treated $(\boldsymbol{C}, \boldsymbol{D})$ animals. In all panels, data are represented as the mean \pm SEM, and bar graphs are also shown as scatter plots.

Ror2 and Ryk contribute to the maintenance of neuropathic pain via regulation of spine plasticity.

Together, these data reveal a specific role of the RTKs, Ror2 and Ryk, in mediating Wnt5a-induced mechanical sensitization and their involvement in the modulation of inflammatory and neuropathic nociceptive hypersensitivity as well as structural plasticity.

\section{Discussion}

In the last years, evidence for a role of Wnt signaling in modulation of the nociceptive pathway has been accumulating (Shi et al., 2012; Zhang et al., 2013; Simonetti et al., 2014; Yuan et al., 2015; Zhu et al., 2018). Wnt signaling was shown to modulate nociception through different mechanisms, both at the level of 
A

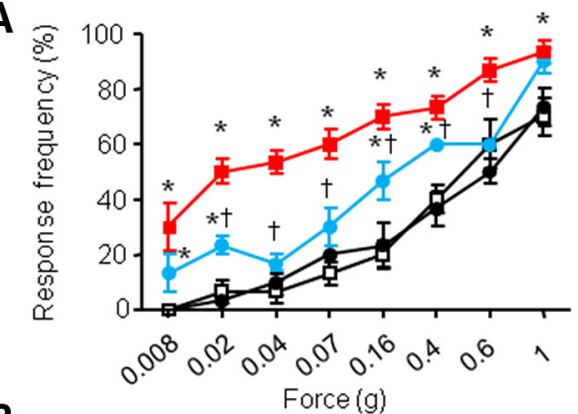

B

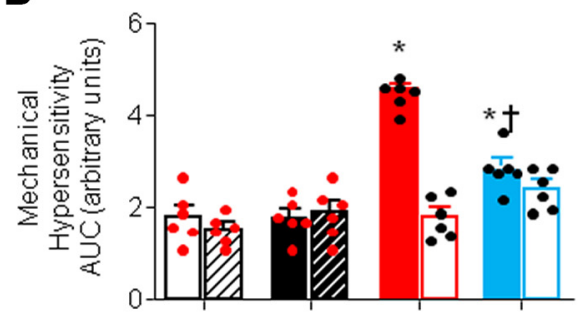

C

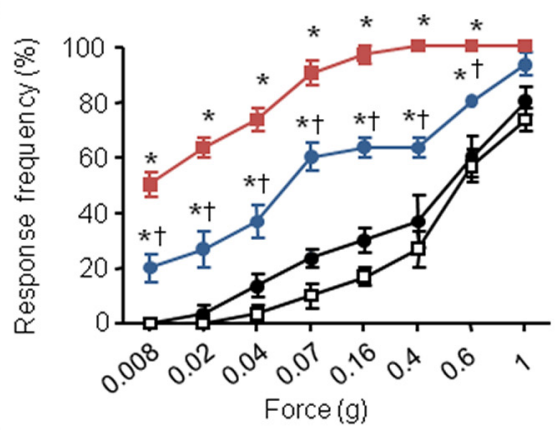

D

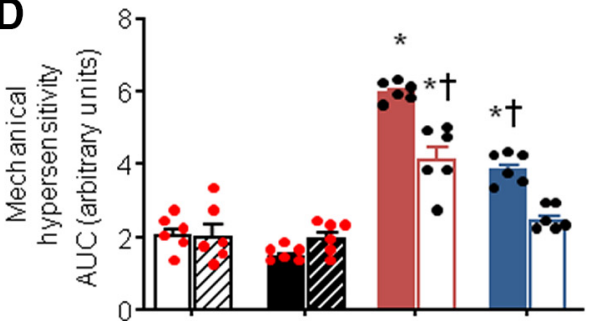

$-\mathbf{- V e h i c l e + s i - S c r a m b l e}$

- Vehicle + si-Ryk

- CFA + si-Scramble

$-C F A+$ si-Ryk

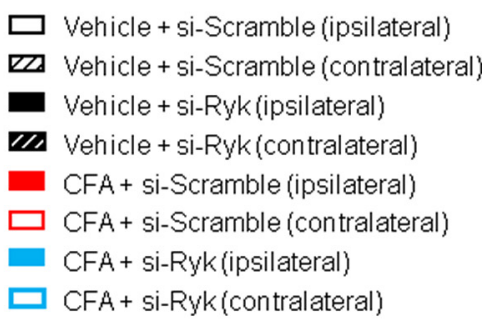

$\square$ Sham + si-Scramble

$\rightarrow$ Sham + si-Ryk

- SNI + si-Scramble

$\bullet$ SNI + si-Ryk

Sham + si-Scramble (ipsilateral)

[7 Sham + si-Scramble (contralateral)

- Sham + si-Ryk (ipsilateral)

II. Sham + si-Ryk (contralateral)

- SNI + si-Scramble (ipsilateral)

$\square$ SNI + si-Scramble (contralateral)

- SNI + si-Ryk (ipsilateral)

$\square \mathrm{SNI}+$ si-Ryk (contralateral)

Figure 7. Spinal Ryk contribute to nociceptive hypersensitivity in mouse models of chronic pain. $\boldsymbol{A}-\boldsymbol{D}$, Behavioral analysis of the effects of intrathecal administration of siRNA against Ryk on mechanical sensitivity $24 \mathrm{~h}$ after intraplantar injection of CFAinduced unilateral paw inflammation $(\boldsymbol{A}, \boldsymbol{B})$ or $7 \mathrm{~d}$ after unilateral nerve injury (SNI; $\boldsymbol{C}, \boldsymbol{D})$. Stimulus-response curves of the ipsilateral paw are shown in $\boldsymbol{A}$ and $\boldsymbol{C}$, whereas the integral over time (AUC) for the inflamed or injured paw (ipsilateral) as well as the contralateral paw are shown in $\boldsymbol{B}$ and $\boldsymbol{D}$. In all panels, $N=8$ mice/group; ANOVA for repeated measures was performed, followed by Tukey's test. ${ }^{*} p<0.05$ compared with the corresponding control group; $t p<0.05$ compared with CFA-treated $(A, B)$ or SNI-treated $(\boldsymbol{C}, \boldsymbol{D})$ animals. In all panels, data are represented as the mean \pm SEM, and bar graphs are also shown as scatter plots.

DRGs as well as spinal cord dorsal horn (Zhang et al., 2013; Itokazu et al., 2014; Simonetti et al., 2014; Liu et al., 2015; Zhu et al., 2018). So far, actions of Wnt signaling at peripheral sensory terminals and the spinal cord were regarded as independent processes and, although rapid functional sensitization processes were described, it remained unclear how Wnt signaling acts in the long term in conditions of inflammatory and neuropathic pain.

Our study, for the first time, causally links Wnt3a signaling in peripheral sensory neurons with $\mathrm{Wnt5a}$ signaling at the spinal cord level in the induction and maintenance of inflammatory and neuropathic hypersensitivity in adult mice. Second, it delivers the novel mechanistic insight that structural plasticity via enhanced

spine morphogenesis in spinal neurons by Wnt5a underlies its role in promoting nociceptive sensitization. Third, it demonstrates that, among the myriad of signaling pathways that can be activated by Wnt signaling, recruitment of the tyrosine kinases Ryk and Ror2 is critical for the modulation of structural plasticity and nociceptive hypersensitivity by Wnt5a, and we go on to show that this process is operational in both inflammatory and neuropathic conditions.

Our previous work has demonstrated that Wnt3a in the periphery is derived primarily from non-neural origin, principally via immune cells in the context of inflammatory pain or from cancer cells in the context of cancer pain (Simonetti et al., 2014). In contrast, we found Wnt5a to be expressed in DRG neurons, and we could demonstrate both ex vivo and in vivo that Wnt3a acting on DRG neurons enhances the release of Wnt5a, suggesting that Wnt5a may be acting in the spinal cord downstream of synaptic release. Interestingly, secreted Wnt have been demonstrated to be transported across synapses via a novel vesicular mechanism of transsynaptic communication in the form of exosomes containing a transmembrane, the conserved protein Evi (Korkut et al., 2009). This suggests that Wnt3a not only sensitizes peripheral neurons, but also further propagates nociceptive sensitization in the spinal cord by triggering the release of Wnt5a at spinal terminals of sensory neurons. We also observed that the long-term pronociceptive effects of peripheral Wnt3a are at least partially dependent on spinal Wnt5a release. These results do not negate, however, that more cellular players can contribute to spinal Wnt5a secretion in vivo. Indeed, recent studies have demonstrated astrocytes as sources of Wnt5a in pathologic conditions, such as peripheral neuropathy and pathologic CNS conditions (Liu et al., 2015; Gonzalez and Rodríguez, 2017).

Wnt5a is emerging to be an important player in the maintenance of different kinds of chronic pain. Wnt5a has been implicated in the pathogenesis of inflammatory pain via the activation of noncanonical pathways in DRG neurons and regulating inflammatory responses (Zhu et al., 2017, 2018; Yuan et al., 2018), as well as in neuropathic pain acting both at the DRG and spinal cord levels (Yuan et al., 2015; Kanda et al., 2016). Our pharmacological data suggest its involvement in the induction phase of inflammatory hypersensitivity, whereas in neuropathy we found Wnt5a to be involved in the maintenance of allodynia. 
However, so far, analyses of both Wnt3a and Wnt5a actions were focused on functional sensitization, while the potential modulation of structural elements was not studied. Here we report that the facilitatory role of Wnt5a in spinal sensitization is associated with dendritic spine remodeling. Activity-dependent morphologic changes in dendritic spines, which form the main cellular substrates for excitatory synaptic contacts, can lead to the potentiation of synaptic transmission, underlining the critical relationship between spine morphology and neuronal function, and providing a structural-based mechanism for modifying long-term synaptic function.

Nerve injury or inflammation has been shown to increase intracellular calcium transients and to induce synaptic potentiation (Sandkühler, 2007; Deumens et al., 2013; Melemedjian et al., 2013, Simonetti et al., 2013). One of the main hallmarks of chronic pain is the central sensitization that can be partially explained by synaptic reorganization in spinal cord pain circuitry (Kuner, 2010). So far, glutamatergic plasticity has been strongly associated with synaptic remodeling in pain states. In inflammatory pain, the continuous flow of nociceptive activity from primary afferents into spinal circuits has been shown to enhance dendritic connectivity in concert with glutamatergic excitability driven by $\mathrm{Ca}^{2+}$ (Simonetti et al., 2013; Lu et al., 2015). There is also evidence for changes in dendritic spine density on nociceptive dorsal horn neurons in injury to the nervous system via trauma, both to peripheral nerves or spinal cord contusion, or metabolic damage in diabetes (Tan et al., 2008, 2012). Our data suggest that, apart from glutamate, Wnt5a released from primary afferent terminals (and potentially astrocytes) also has the ability to promote the buildup of dendritic spines, thereby increasing excitation in a sustained manner. This is not only valid when applied exogenously, but importantly, we observed that blocking Wnt5a signaling leads to a reduction of the CFA- or SNI-induced increase of dendritic spine density in dorsal horn neurons and reduced behavioral hypersensitivity in the same animals. This indicates that Wnt5a-mediated spine remodeling is recruited endogenously as a key step in mediating nociceptive hypersensitivity in neuropathic and inflammatory pain conditions. Given that several components of the Wnt signaling pathway were reported to be regulated spinally in other neurologic conditions, such as spinal cord injury (González-Fernández et al., 2014), it will be interesting to determine in further studies whether Wnt signaling alters spinal connectivity in these pathologies as well.

Developmentally, Wnt signaling has been implicated in synaptogenesis (Salinas, 1999), which has been attributed to different
Wnt ligands and several type of Wnt receptors, such as Frizzled 5 and Frizzled 9 (Farías et al., 2009; Ciani et al., 2011; Hiester et al., 2013; Stamatakou et al., 2013). In particular, Wnt7a stimulates excitatory synapse formation and function via CaMKIIdependent recruitment of PSD-95 and spine growth (Ciani et al., 2011). Likewise, Wnt5a was shown to induce JNK-dependent PSD clustering (Farías et al., 2009), thereby modulating the differentiation of synapses (Farías et al., 2009; Cuitino et al., 2010; Varela-Nallar et al., 2010). Moreover, Wnt5a has been described to functionally regulate synaptic transmission via modulation of NMDA receptor-mediated synaptic transmission (Cerpa et al., 2011). That this is also relevant in the context of pain is shown by studies reporting that at the functional level, Wnt/Ryk signaling modulates spinal excitability in neuropathic rats (Liu et al., 2015). However, the mechanisms via which synaptic transmission and molecules involved in synaptic processing are altered were not clear. Our results on structural remodeling of dendritic spines by Wnt5a signaling now indicate that the formation of new synaptic contacts may provide the mechanistic basis for enhanced synaptic transmission and potentiation.

Previous studies on synaptic spine remodeling in spinal circuits in chronic pain states uncovered the RhoGTAPse Rac 1 as a major mechanistic mediator (Tan et al., 2012). Racl is an important regulator of the actin cytoskeleton and rapid actin rearrangement is key to enabling structural spine remodeling 
(Nakayama and Luo, 2000; Kuner and Flor, 2016) However, how Rac1 is intracellularly recruited in spinal neurons in states of chronic pain was puzzling. Recently, Lu et al. (2015) showed that a synaptic protein, Kalirin-7, forms a molecular bridge linking synaptic glutamatergic receptor signaling to Racl activation. Here, our results suggest that Wnt5a release may represent a parallel pathway to glutamatergic signaling for eliciting structural changes at synapses between primary afferents and spinal neurons and, second, that it does so by recruiting Ryk and Ror2 kinases. Wnt5a has been reported to stimulate Racl activation through the PCP (planar cell polarity) pathway in some cell types (Lutze et al., 2019), suggesting that Ryk1 and Ror2 acting as coreceptors may play a role. So far, however, clear links between Ryk activation and Racl signaling have not been described. There is one study reporting that Ror 1 and Ror2 are required for Wnt5ainduced activation and internalization of the Frizzled 2 receptor and subsequent noncanonical activation of Rac1 in a laboratory HeLaS3 cell line (Sato et al., 2010), but studies performed in native neurons are lacking. Thus, it remains unclear whether Rac1 and Ryk/Ror2 signaling act in series or in parallel. A concerted action of these two signaling pathways is plausible because the effects of downregulation or inhibition of these pathways on pain-associated spine remodeling are mostly partial. Further studies are needed to test the potential links between Rac1 and Ryk/Ror2 signaling downstream of Wnt5a activation in models of pathologic pain.

Although siRNA-mediated knockdown of Ryk and Ror2 expression in the spinal cord abolished spine remodeling in chronic pain conditions, it is important to acknowledge that the study does not conclusively establish whether spine remodeling occurs in those spinal neurons that express Ryk or Ror2 or whether this is a secondary effect downstream of Ryk/Ror2 expressed in other neurons or glia via paracrine signaling. Technical limitations hinder us from establishing this link. Ryk and Ror2 are expressed widely in spinal neurons in chronic pain states (Liu et al., 2015; Zhou et al., 2019), and are particularly broadly found across the neuropil, rendering it likely that these receptors are indeed expressed on neurons undergoing remodeling in chronic pain states. Our data on localization of these receptors to synaptic markers further render this likely. Future studies addressing these diverse scenarios will be insightful.

Several observations indicate that Ryk and Ror2 are very well placed to mediate structural plasticity in nociceptive pathways. Ryk is reported to be expressed in sensory and spinal neurons and to be upregulated after nerve injury (Yang et al., 2017). Ryk activity has been previously linked to phenotypic effects of Wnt signaling on neuronal morphology in the developing and regenerating nervous system (Fradkin et al., 2010), in particular with respect to neurite outgrowth and axon guidance (Lu et al., 2004; Paganoni and Ferreira, 2005; Keeble et al., 2006). In cultured hippocampal neurons, Ror2 is reported to regulate developmental spine morphogenesis (Paganoni et al., 2010) and found to be necessary to maintain dendritic spine number and distribution in adult neurons (Alfaro et al., 2015). Mice lacking Ror2 exhibit abnormalities that mirror phenotypes observed in Wnt5a-null mutant mice (Ho et al., 2012). Here we found that both Ryk and Ror2 are required for normal development of neuropathic and inflammatory hypersensitivity as well as for spine remodeling in the spinal dorsal horn. Since these receptor tyrosine kinases can activate the same intracellular effectors and both form complexes with
Frizzled receptors ( $\mathrm{Lu}$ et al.,2004; Nishita et al., 2010), it is possible that their corecruitment enables a strong, synergistic activation of intracellular signaling cascade components and thereby modulates dendritic spine remodeling in the nociceptive pathway. Alternatively, it cannot be ruled out that Ryk and Ror2 form functional heterodimers in spinal neurons, although there are no reports for that in the literature so far. Moreover, it is important to bear in mind that the diverse mediators of Wnt signaling are expressed in neurons as well as glia cells in the spinal cord dorsal horn and could therefore be acting in concert to regulate nociceptive sensitivity by acting via different types of cells. Further studies are needed to explore these possibilities and to characterize the precise orchestration of molecular pathways involved in Wnt-mediated spine remodeling in the nociceptive system. Finally, it is also plausible that the activation of Ryk and Ror2 may be triggering diverse downstream pathways outside the scope of well described classical Wnt signaling. For example, Ryk has been recently reported to enhance the release of CCL2, thereby modulating spinal nerve ligation-induced mechanical hyperalgesia (Yang et al., 2017).

In conclusion, we report a novel pathway comprising Wnt3ainduced Wnt5a release in the spinal dorsal horn following nociceptive stimulation. We report that Wnt5a, via Ryk/Ror2 signaling, has the ability to induce structural remodeling of spinal circuits in inflammatory and neuropathic pain, which contributes to amplifying nociceptive sensitivity. Furthermore, this study provides proof-of-concept for targeting spinal Wnt5a/ Ryk/Ror2 signaling for alleviating nociceptive hypersensitivity in vivo.

\section{References}

Alfaro IE, Varela-Nallar L, Varas-Godoy M, Inestrosa NC (2015) The ROR2 tyrosine kinase receptor regulates dendritic spine morphogenesis in hippocampal neurons. Mol Cell Neurosci 67:22-30.

Budnik V, Salinas PC (2011) Wnt signaling during synaptic development and plasticity. Curr Opin Neurobiol 21:151-159.

Cerpa W, Gambrill A, Inestrosa NC, Barria A (2011) Regulation of NMDAreceptor synaptic transmission by Wnt signaling. J Neurosci 31:94669471.

Chen J, Park CS, Tang SJ (2006) Activity-dependent synaptic Wnt release regulates hippocampal long term potentiation. J Biol Chem 281:1191011916.

Ciani L, Boyle KA, Dickins E, Sahores M, Anane D, Lopes DM, Gibb AJ, Salinas PC (2011) Wnt7a signaling promotes dendritic spine growth and synaptic strength through $\mathrm{Ca}^{2+} /$ calmodulin-dependent protein kinase II. Proc Natl Acad Sci U S A 108:10732-10737.

Cuitino L, Godoy JA, Farías GG, Couve A, Bonansco C, Fuenzalida M, Inestrosa $\mathrm{NC}$ (2010) Wnt-5a modulates recycling of functional $\mathrm{GABA}_{\mathrm{A}}$ receptors on hippocampal neurons. J Neurosci 30:8411-8420.

Decosterd I, Allchorne A, Woolf CJ (2002) Progressive tactile hypersensitivity after a peripheral nerve crush: non-noxious mechanical stimulusinduced neuropathic pain. Pain 100:155-162.

Deumens R, Mazzone GL, Taccola G (2013) Early spread of hyperexcitability to caudal dorsal horn networks after a chemically-induced lesion of the rat spinal cord in vitro. Neuroscience 229:155-163.

Farías GG, Alfaro IE, Cerpa W, Grabowski CP, Godoy JA, Bonansco C, Inestrosa NC (2009) Wnt-5a/JNK signaling promotes the clustering of PSD-95 in hippocampal neurons. J Biol Chem 284:15857-15866.

Fradkin LG, Dura JM, Noordermeer JN (2010) Ryks: new partners for Wnts in the developing and regenerating nervous system. Trends Neurosci 33:84-92.

Gangadharan V, Wang R, Ulzhöfer B, Luo C, Bardoni R, Bali KK, Agarwal N, Tegeder I, Hildebrandt U, Nagy GG, Todd AJ, Ghirri A, Häussler A, Sprengel R, Seeburg PH, MacDermott AB, Lewin GR, Kuner R (2011) Peripheral calcium-permeable AMPA receptors regulate chronic inflammatory pain in mice. J Clin Invest 121:1608-1623. 
González-Fernández C, Fernández-Martos CM, Shields SD, Arenas E, Javier Rodríguez F (2014) Wnts are expressed in the spinal cord of adult mice and are differentially induced after injury. J Neurotrauma 31 : 565-581.

Gonzalez P, Rodríguez FJ (2017) Analysis of the expression of the Wnt family of proteins and its modulatory role on cytokine expression in non activated and activated astroglial cells. Neurosci Res 114:16-29.

Hartmann B, Ahmadi S, Heppenstall PA, Lewin GR, Schott C, Borchardt T, Seeburg PH, Zeilhofer HU, Sprengel R, Kuner R (2004) The AMPA receptor subunits GluR-A and GluR-B reciprocally modulate spinal synaptic plasticity and inflammatory pain. Neuron 44:637-650.

Hiester BG, Galati DF, Salinas PC, Jones KR (2013) Neurotrophin and Wnt signaling cooperatively regulate dendritic spine formation. Mol Cell Neurosci 56:115-127.

Ho HY, Susman MW, Bikoff JB, Ryu YK, Jonas AM, Hu L, Kuruvilla R, Greenberg ME (2012) Wnt5a-Ror-Dishevelled signaling constitutes a core developmental pathway that controls tissue morphogenesis. Proc Natl Acad Sci U S A 109:4044-4051.

Inestrosa NC, Montecinos-Oliva C, Fuenzalida M (2012) Wnt signaling: role in Alzheimer disease and schizophrenia. J Neuroimmune Pharmacol 7:788-807.

Itokazu T, Hayano Y, Takahashi R, Yamashita T (2014) Involvement of Wnt/ $\beta$-catenin signaling in the development of neuropathic pain. Neurosci Res 79:34-40.

Jenei V, Sherwood V, Howlin J, Linnskog R, Säfholm A, Axelsson L, Andersson T (2009) A t-butyloxycarbonyl-modified Wnt5a-derived hexapeptide functions as a potent antagonist of Wnt5a-dependent melanoma cell invasion. Proc Natl Acad Sci U S A 106:19473-19478.

Kanda H, Kanao M, Liu S, Yi H, Iida T, Levitt RC, Candiotti KA, Lubarsky DA, Hao S (2016) HSV vector-mediated GAD67 suppresses neuropathic pain induced by perineural HIV gp120 in rats through inhibition of ROS and Wnt5a. Gene Ther 23:340-348.

Keeble TR, Halford MM, Seaman C, Kee N, Macheda M, Anderson RB, Stacker SA, Cooper HM (2006) The Wnt receptor Ryk is required for Wnt5a-mediated axon guidance on the contralateral side of the corpus callosum. J Neurosci 26:5840-5848.

Kikuchi A, Yamamoto H, Sato A, Matsumoto S (2012) Wnt5a: its signalling, functions and implication in diseases. Acta Physiol (Oxf) 204:1733

Korkut C, Ataman B, Ramachandran P, Ashley J, Barria R, Gherbesi N, Budnik V (2009) Trans-synaptic transmission of vesicular Wnt signals through Evi/Wntless. Cell 139:393-404.

Kuner R (2010) Central mechanisms of pathological pain. Nat Med 16:12581266.

Kuner R, Flor H (2016) Structural plasticity and reorganisation in chronic pain. Nat Rev Neurosci 18:20-30.

Liu S, Liu YP, Huang ZJ, Zhang YK, Song AA, Ma PC, Song XJ (2015) Wnt/ Ryk signaling contributes to neuropathic pain by regulating sensory neuron excitability and spinal synaptic plasticity in rats. Pain 156: 2572-2584.

Lu J, Luo C, Bali KK, Xie RG, Mains RE, Eipper BA, Kuner R (2015) A role for Kalirin-7 in nociceptive sensitization via activity-dependent modulation of spinal synapses. Nat Commun 6:6820.

Lu W, Yamamoto V, Ortega B, Baltimore D (2004) Mammalian Ryk is a Wnt coreceptor required for stimulation of neurite outgrowth. Cell 119:97-108

Lutze G, Haarmann A, Demanou Toukam JA, Buttler K, Wilting J, Becker J (2019) Non-canonical WNT-signaling controls differentiation of lymphatics and extension lymphangiogenesis via RAC and JNK signaling. Sci Rep 9:4739.

Melemedjian OK, Tillu DV, Asiedu MN, Mandell EK, Moy JK, Blute VM, Taylor CJ, Ghosh S, Price TJ (2013) BDNF regulates atypical PKC at spinal synapses to initiate and maintain a centralized chronic pain state. Mol Pain 9:12.

Nakayama AY, Luo L (2000) Intracellular signaling pathways that regulate dendritic spine morphogenesis. Hippocampus 10:582-586.

Niehrs C (2012) The complex world of WNT receptor signalling. Nat Rev Mol Cell Biol 13:767-779.

Nishita M, Itsukushima S, Nomachi A, Endo M, Wang Z, Inaba D, Qiao S, Takada S, Kikuchi A, Minami Y (2010) Ror2/Frizzled complex mediates Wnt5a-induced AP-1 activation by regulating Dishevelled polymerization. Mol Cell Biol 30:3610-3619.
Njoo C, Heinl C, Kuner R (2014) In vivo SiRNA transfection and gene knockdown in spinal cord via rapid noninvasive lumbar intrathecal injections in mice. J Vis $\operatorname{Exp}(85): 51229$.

Oliva CA, Vargas JY, Inestrosa NC (2013a) Wnt signaling: role in LTP, neural networks and memory. Ageing Res Rev 12:786-800.

Oliva CA, Vargas JY, Inestrosa NC (2013b) Wnts in adult brain: from synaptic plasticity to cognitive deficiencies. Front Cell Neurosci 7:224.

Paganoni S, Ferreira A (2005) Neurite extension in central neurons: a novel role for the receptor tyrosine kinases Ror1 and Ror2. J Cell Sci 118:433446.

Paganoni S, Bernstein J, Ferreira A (2010) Ror1-Ror2 complexes modulate synapse formation in hippocampal neurons. Neuroscience 165:12611274.

Patapoutian A, Reichardt LF (2000) Roles of Wnt proteins in neural development and maintenance. Curr Opin Neurobiol 10:392-399.

Salinas PC (1999) Wnt factors in axonal remodelling and synaptogenesis. Biochem Soc Symp 65:101-109.

Sandkühler J (2007) Understanding LTP in pain pathways. Mol Pain 3:9.

Sato A, Yamamoto H, Sakane H, Koyama H, Kikuchi A (2010) Wnt5a regulates distinct signalling pathways by binding to Frizzled2. EMBO J 29: 41-54.

Schweizerhof M, Stösser S, Kurejova M, Njoo C, Gangadharan V, Agarwal N, Schmelz M, Bali KK, Michalski CW, Brugger S, Dickenson A, Simone DA, Kuner R (2009) Hematopoietic colony-stimulating factors mediate tumor-nerve interactions and bone cancer pain. Nat Med 15:802807.

Shi Y, Yuan S, Li B, Wang J, Carlton SM, Chung K, Chung JM, Tang SJ (2012) Regulation of Wnt signaling by nociceptive input in animal models. Mol Pain 8:47.

Simonetti M, Hagenston AM, Vardeh D, Freitag HE, Mauceri D, Lu J, Satagopam VP, Schneider R, Costigan M, Bading H, Kuner R (2013) Nuclear calcium signaling in spinal neurons drives a genomic program required for persistent inflammatory pain. Neuron 77:43-57.

Simonetti M, Agarwal N, Stösser S, Bali KK, Karaulanov E, Kamble R, Pospisilova B, Kurejova M, Birchmeier W, Niehrs C, Heppenstall P, Kuner R (2014) Wnt-Fzd signaling sensitizes peripheral sensory neurons via distinct noncanonical pathways. Neuron 83:104-121.

Stamatakou E, Marzo A, Gibb A, Salinas PC (2013) Activity-dependent spine morphogenesis: a role for the actin-capping protein Eps8. J Neurosci 33:2661-2670.

Stösser S, Agarwal N, Tappe-Theodor A, Yanagisawa M, Kuner R (2010) Dissecting the functional significance of endothelin A receptors in peripheral nociceptors in vivo via conditional gene deletion. Pain 148: 206-214.

Tan AM, Waxman SG (2015) Dendritic spine dysgenesis in neuropathic pain. Neurosci Lett 601:54-60.

Tan AM, Stamboulian S, Chang Y-W, Zhao P, Hains AB, Waxman SG, Hains BC (2008) Neuropathic pain memory is maintained by Rac1-regulated dendritic spine remodeling after spinal cord injury. J Neurosci 28:13173-13183.

Tan AM, Chang YW, Zhao P, Hains BC, Waxman SG (2011) Rac1-regulated dendritic spine remodeling contributes to neuropathic pain after peripheral nerve injury. Exp Neurol 232:222-233.

Tan AM, Samad OA, Fischer TZ, Zhao P, Persson AK, Waxman SG (2012) Maladaptive dendritic spine remodeling contributes to diabetic neuropathic pain. J Neurosci 32:6795-6807.

Tappe A, Klugmann M, Luo C, Hirlinger D, Agarwal N, Benrath J, Ehrengruber MU, During MJ, Kuner R (2006) Synaptic scaffolding protein Homerla protects against chronic inflammatory pain. Nat Med 12:677-681.

Varela-Nallar L, Alfaro IE, Serrano FG, Parodi J, Inestrosa NC (2010) Wingless-type family member $5 \mathrm{~A}$ (Wnt-5a) stimulates synaptic differentiation and function of glutamatergic synapses. Proc Natl Acad Sci U S A 107:21164-21169.

Wang J, Zhang S, Li L, Zhang L (2015) Involvement of Wnt5a within the cerebrospinal fluid-contacting nucleus in nerve injury-induced neuropathic pain. Int J Neurosci 125:147-153.

Yamamoto S, Nishimura O, Misaki K, Nishita M, Minami Y, Yonemura S, Tarui H, Sasaki H (2008) Cthrcl selectively activates the planar cell polarity pathway of Wnt signaling by stabilizing the Wnt-receptor complex. Dev Cell 15:23-36. 
Yang QO, Yang WJ, Li J, Liu FT, Yuan H, Ou Yang YP (2017) Ryk receptors on unmyelinated nerve fibers mediate excitatory synaptic transmission and CCL2 release during neuropathic pain induced by peripheral nerve injury. Mol Pain 13:1744806917709372.

Yuan S, Shi Y, Tang SJ (2012) Wnt signaling in the pathogenesis of multiple sclerosis-associated chronic pain. J Neuroimmune Pharmacol 7:904913.

Yuan SB, Ji G, Li B, Andersson T, Neugebauer V, Tang SJ (2015) A Wnt5a signaling pathway in the pathogenesis of HIV-1 gp120-induced pain. Pain 156:1311-1319.

Yuan S, Shi Y, Guo K, Tang SJ (2018) Nucleoside reverse transcriptase inhibitors (NRTIs) induce pathological pain through Wnt5a-mediated neuroinflammation in aging mice. J Neuroimmune Pharmacol 13:230236.
Zhang YK, Huang ZJ, Liu S, Liu YP, Song AA, Song XJ (2013) WNT signaling underlies the pathogenesis of neuropathic pain in rodents. J Clin Invest 123:2268-2286.

Zhu A, Shen L, Xu L, Chen W, Huang Y (2017) Suppression of Wnt5a, but not Wnts, relieves chronic post-thoracotomy pain via anti-inflammatory modulation in rats. Biochem Biophys Res Commun 493:474-480.

Zhu A, Shen L, Xu L, Chen W, Huang Y (2018) Wnt5a mediates chronic post-thoracotomy pain by regulating non-canonical pathways, nerve regeneration, and inflammation in rats. Cell Signal 44:51-61.

Zhou XL, Zhang CJ, Peng YN, Wang Y, Xu HJ, Liu CM (2019) ROR2 modulates neuropathic pain via phosphorylation of NMDA receptor subunit GluN2B in rats. Br J Anaesth 123:e239-e248.

Zimmerman ZF, Moon RT, Chien AJ (2012) Targeting Wnt pathways in disease. Cold Spring Harb Perspect Biol 4:a008086. 